\title{
ACTIONS OF MULTIPLIER HOPF ALGEBRAS
}

\author{
Bernhard Drabant, Alfons Van Daele and Yinhuo Zhang
}

\author{
April 1997
}

\begin{abstract}
For an action $\alpha$ of a group $G$ on an algebra $R$ (over $\mathbb{C}$ ), the crossed product $R \times{ }_{\alpha} G$ is the vector space of $R$-valued functions with finite support in $G$, together with the twisted convolution product given by
\end{abstract}

$$
(\xi \eta)(p)=\sum_{q \in G} \xi(q) \alpha_{q}\left(\eta\left(q^{-1} p\right)\right)
$$

where $p \in G$. This construction has been extended to the theory of Hopf algebras. Given an action of a Hopf algebra $A$ on an algebra $R$, it is possible to make the tensor product $R \otimes A$ into an algebra by using a twisted product, involving the action. In this case, the algebra is called the smash product and denoted by $R \# A$. In the group case, the action $\alpha$ of $G$ on $R$ yields an action of the group algebra $\mathbb{C} G$ as a Hopf algebra on $R$ and the crossed $R \times{ }_{\alpha} G$ coincides with the smash product $R \# \mathbb{C} G$.

In this paper we extend the theory of actions of Hopf algebras to actions of multiplier Hopf algebras. We also construct the smash product and we obtain results very similar as in the original situation for Hopf algebras.

The main result in the paper is a duality theorem for such actions. We consider dual pairs of multiplier Hopf algebras to formulate this duality theorem. We prove a result in the case of an algebraic quantum group and its dual. The more general case is only stated and will be proven in a separate paper on coactions. These duality theorems for actions are substantial generalizations of the corresponding theorem for Hopf algebras. Also the techniques that are used here to prove this result are slightly different and simpler.

\section{INTRODUCTION}

Let $R$ be an algebra over $\mathbb{C}$ with identity and let $G$ be a group. Assume that $\alpha$ is an action of $G$ on $R$ by means of automorphisms of $R$. So, for every $p \in G$ we have an automorphism $\alpha_{p}$ of $R$ and we also have $\alpha_{p q}(x)=\alpha_{p}\left(\alpha_{q}(x)\right)$ for all $x \in R$ and $p, q \in G$. It follows that $\alpha_{e}(x)=x$ for all $x \in R$ when $e$ is the identity of $G$. There is a standard procedure to associate a new algebra $R \times{ }_{\alpha} G$ to such a triple $(R, G, \alpha)$. The elements in this algebra are $R$-valued functions with finite support

\footnotetext{
E-mail: b.drabant@damtp.cam.ac.uk alfons.vandaele@wis.kuleuven.ac.be zhang@davinci.wis.kuleuven.ac.be
} 
in $G$. This set is made into a complex vector space in the obvious way and the product of two such functions $\xi, \eta$ is a twisted convolution product, given by

$$
(\xi \eta)(p)=\sum_{q \in G} \xi(q) \alpha_{q}\left(\eta\left(q^{-1} p\right)\right)
$$

whenever $p \in G$. This new algebra $R \times{ }_{\alpha} G$ is called the crossed product or semidirect product of $R$ with the action $\alpha$ of $G$. In the trivial case where $R=\mathbb{C}$, and so where $G$ acts trivially on $\mathbb{C}$, we get of course the group algebra $\mathbb{C} G$ of $G$ over $\mathbb{C}$. If $R$ is non-trivial, but if the action is still trivial, it is clear that the crossed product is the tensor product of $R$ with the group algebra $\mathbb{C} G$. If fact, if the action of $G$ on $R$ is inner, we still get an algebra, isomorphic with this tensor product algebra. When $R$ is itself the group algebra $\mathbb{C} H$ of a group $H$ and when $\alpha$ is coming from an action of $G$ on $H$, then the crossed product is the group algebra of the semi-direct product of $G$ and $H$. In the general case, this crossed product construction provides examples of non-trivial algebras. Moreover, some nice properties can be proven. One of them is the duality for such crossed products (see e.g. [C-M] and $[\mathrm{Q}]$ ). We will say more about this further in the introduction.

The above construction has been generalized to actions of Hopf algebras. This is done as follows. Again let $R$ be an algebra over $\mathbb{C}$ with identity. Let $A$ be a Hopf algebra over $\mathbb{C}$. By an action of $A$ on $R$ is meant a bilinear map $a \otimes x \rightarrow a x$ from $A \otimes R$ to $R$ such that $R$ is a left $A$-module and such that

$$
a(x y)=\sum\left(a_{(1)} x\right)\left(a_{(2)} y\right)
$$

for all $x, y \in R$ and $a \in A$ (we use the Sweedler convention). Here, it is also natural to require that $1 x=x$ for all $x \in R$ where 1 is the identity in $A$. Again, a new algebra can be constructed. In this case, the tensor product $R \otimes A$ is the underlying vector space over $\mathbb{C}$ while the product is again a twisted product given by

$$
(x \otimes a)(y \otimes b)=\sum x\left(a_{(1)} y\right) \otimes a_{(2)} b
$$

for all $a, b \in A$ and $x, y \in R$. In this setting, the new algebra is called the smash product (rather than the crossed product) and it is denoted by $R \# A$. (We will discuss the terminology later in this introduction). Also here, this construction provides interesting examples and nice results can be proven (see e.g. [M2]).

When $G$ is a group, acting on an algebra $R$ and when $A$ is the Hopf algebra $\mathbb{C} G$, then it is quite straightforward to see that $A$ acts on $R$ by means of the map

$$
f \otimes x \rightarrow \sum f(p) \alpha_{p}(x)
$$

where $x \in R$ and $f$ is a complex function with finite support in $G$ (considered as an element in $\mathbb{C} G$ ). It is also easy to check that the crossed product of $R$ by the action $\alpha$ of $G$, as defined before, coincides with the smash product of $R$ with $A$ is this case. So, indeed, the smash product for an action of a Hopf algebra extends the notion of the crossed (or semi-direct) product for an action of a group. 
One of the main results in the theory of smash products is the duality. Let us formulate this in its simplest form. We consider a finite-dimensional Hopf algebra $A$ and its dual Hopf algebra $A^{\prime}$. As before, let $A$ act on $R$ and consider the smash product $R \# A$. The dual action of $A^{\prime}$ on $R \# A$ is now defined by

$$
\omega(x \# a)=\sum \omega\left(a_{(2)}\right) x \# a_{(1)}
$$

whenever $x \in R, a \in A$ and $\omega \in A^{\prime}$. It is possible to look at the bismash product $(R \# A) \# A^{\prime}$ and it turns out that this algebra is isomorphic with the algebra $M_{n}(R)$ of $n \times n$ matrices over $R$ where $n$ is the dimension of $A$. It is remarkable that the bismash product no longer depends on the underlying Hopf algebra, nor on the action. A similar result still holds in more general situations (see [M2]).

This duality is an extension of the duality for crossed products of actions of finite abelian groups. In this case, the dual action is an action of the dual group. The duality is related with the Pontryagin duality between a group $G$ and its dual group $\hat{G}$. This theory for group actions has been extended in various directions. One of the fundamental results has been obtained in the von Neumann algebra case. There, a locally compact abelian group $G$ acts on a von Neumann algebra $M$ in a continuous way and the crossed product $M \times{ }_{\alpha} G$ is a von Neumann algebra, constructed in a way very similar to the case described above. The Pontryagin dual is now again an abelian locally compact group $\hat{G}$ and the dual action $\hat{\alpha}$ is a natural action of $\hat{G}$ on the crossed product $M \times_{\alpha} G$. The double crossed product $\left(M \times_{\alpha} G\right) \times_{\hat{\alpha}} \hat{G}$ turns out to be canonically isomorphic with the von Neumann algebra tensor product $M \bar{\otimes} \mathcal{B}\left(\mathcal{L}^{2}(G)\right)$ of the original von Neumann algebra $M$ with the algebra of all bounded linear operators on the Hilbert space $\mathcal{L}^{2}(G)$ of all square integrable functions w.r.t. a left Haar measure on $G$.

This duality for actions of locally compact groups on von Neumann algebras was in fact the first duality of this kind that was obtained and the duality for actions of Hopf algebras was inspired by this result. It was proven by Takesaki [Ts] (see also $[\mathrm{N}-\mathrm{T}]$ and [VD1]).

But this result has also been generalized within the field of operator algebras in various directions. First, there is the duality for actions of locally compact groups on $\mathrm{C}^{*}$-algebras $[\mathrm{T}]$, see aslo $[\mathrm{P}]$. There are also different versions for non-abelian groups. Different aspects of non-abelian abstract harmonic analysis come in. One of the more sophisticated results is the duality for actions of Kac algebras on von Neumann algebras [E-S]. In the operator algebra context, it seems more natural to work with coactions, rather than with actions. In fact recently, some work has been obtained for coactions of Hopf $\mathrm{C}^{*}$-algebras $[\mathrm{Ng}]$.

All this work points in the direction of locally compact quantum groups. Unfortunately, there still is no satisfactory definition of such a locally compact quantum 
group. Recently, there has been some progress in developing such a notion. We have the work of Masuda, Nakagami and Woronowicz ([M-N] and [M-N-W]). There is also the theory, developed by one of the authors on multiplier Hopf algebras and algebraic quantum groups [VD5, VD6]. The $\mathrm{C}^{*}$-version can be found in [K-VD] and $[K]$. The theory of algebraic quantum groups is not so general as the theory of Masuda and his coauthors. On the other hand, it is more accessable and still it contains many interesting cases, such as the discrete and the compact quantum groups. It also behaves well with respect to duality.

Because of this, and because this theory of algebraic quantum groups is close in nature to the theory of Hopf algebras, it is quite natural to look at the theory of actions of Hopf algebras and to try to extend it to the multiplier Hopf algebras. This is in fact the content of this paper.

When a Hopf algebra $A$ is infinite-dimensional, the space $A^{\prime}$ of all linear functionals is still an algebra, but no longer a Hopf algebra. The natural candidate for the comultiplicaton on $A^{\prime}$ will have values in $(A \otimes A)^{\prime}$ but this space is strictly bigger than $A^{\prime} \otimes A^{\prime}$. In some cases, this problem can be overcome by taking the subspace $A^{0}$ of elements in $A^{\prime}$ for which the image of the comultiplication lies in $A^{\prime} \otimes A^{\prime}$. This is a Hopf algebra, but unfortunately, in general, it may be very small. When it is big enough, we are essentially dealing with a (non-degenerate) dual pair of Hopf algebras. The setting of dual pairs is a natural framework to formulate results that are easy to obtain for the finite-dimensional case (because $A^{\prime}$ is again a Hopf algebra), and cannot be generalized to arbitrary Hopf algebras.

A typical such result is the construction of the quantum double by Drinfel'd [D] (see also [VD2]). Also the duality for actions is a result of this nature (see [M2]).

Recently, the theory of Hopf algebras has been generalized to the so-called multiplier Hopf algebras and also the dual pairs of such multiplier Hopf algebras have been studied [D-VD]. The quantum double could be constructed for such dual pairs. And it should be no surprise that also the theory of actions and the duality can be generalized to multiplier Hopf algebras and dual pairs of multiplier Hopf algebras.

In section 2, we first recall the notion of a multiplier Hopf algebra. Very roughly speaking, it is the natural extension of the notion of a Hopf algebra where the underlying algebra is no longer assumed to have an identity. It has been argued in previous papers (see e.g. [VD3] and [VD6]) why such an extension is important. Apart from recalling the definition of a multiplier Hopf algebra, we also prove some new results for general multiplier Hopf algebras (existence of local units), important as such, but especially nice for dealing with modules further in the paper. In section 2, we also recall some special cases of multiplier Hopf algebras (with the existence of integrals and cointegrals). 
In section 3, we treat modules. Multiplier Hopf algebra modules where already considered in $[\mathrm{D}-\mathrm{VD}]$. Here we investigate them closer. We have special attention for the features that are new because our algebras no longer are assumed to have an identity. The natural condition $1 x=x$ for a module over an algebra with identity will be replaced by the condition $A R=R$ (where $A$ is an algebra and $R$ a left $A$-module). We will explain where this condition comes from and why it is natural. We call such a module unital.

In section 4, we consider actions of a multiplier Hopf algebra $A$ on an algebra $R$ and define the notion of an $A$-module algebra. To begin with, $R$ is a unital left $A$-module. Moreover, we require that also

$$
a(x y)=m(\Delta(a)(x \otimes y))
$$

whenever $x, y \in R$ and $a \in A$ (and where $m$ denotes multiplication as a linear map from $R \otimes R$ to $R$ ). Precisely, because the module $R$ is assumed to be unital, we can think of elements $y$ as elements of the form $b z$ where $b \in A$ and $z \in R$ and therefore the above right hand side can be read as

$$
m((\Delta(a)(1 \otimes b))(x \otimes z))
$$

and for multiplier Hopf algebras, we do have that $\Delta(A)(1 \otimes A) \subseteq A \otimes A$.

In section 4, we also consider the adjoint action and related notions such as inner actions, cocycle equivalent actions,... One can define the fixed point algebra within $R$ but it turns out to be more natural first to extend the action of $A$ to the multiplier algebra $M(R)$ and then to consider fixed points in $M(R)$. Also this is treated in the fourth section.

In section 5, we construct the smash product for an action of a multiplier Hopf algebra $A$ on an algebra $R$. It will be denoted by $R \# A$. We obtain some natural properties. We get a universal property characterizing $R \# A$. We obtain that $R \# A$ is the tensor product algebra $R \otimes A$ when the action is inner and we find that cocycle equivalent actions yield isomorphic smash products.

The main section is section 7 where we obtain our duality results. However, we first need to study some special cases of the previous theory. In all the previous sections, we have used the case of a group action as a motivating example. In section 6 however, we treat the less trivial examples. The basic framework is that of a dual pair of multiplier Hopf algebras as introduced in [D-VD]. Moreover, we relate the results of this paper with the constructions needed to obtain the quantum double. As a special case, we have the pairing of an algebraic quantum group $A$ with its dual $\hat{A}$ as in [VD5, VD6]. This case is closest to the duality of finite-dimensional Hopf algebras. But it is already far more general. 
The duality theorem in section 7 is formulated in the same framework of dual pairs. However, it turns out that the conditions we need to prove this theorem for arbitrary dual pairs makes the result in fact a theorem about coactions. Since we are preparing a separate paper on coactions of multiplier Hopf algebras ([VD-Z3]), we will not give the proof of the general result here. For algebraic quantum groups, the result is nicer and easier to proof. The conditions that we need in the more general case turn out to be automatic for algebraic quantum groups. So, the duality theorem that we obtain here for algebraic quantum groups is really a special case of the one obtained in [VD-Z3] for more general pairings. We will explain all this in this last section.

This more restricted duality (in the case of algebraic quantum groups) turns out to be very similar to the original duality results proven in the theory of actions of locally compact groups on von Neumann algebras and $\mathrm{C}^{*}$-algebras.

Let us now fix some terminology, notations and standard references.

The standard references for Hopf algebras are $[\mathrm{A}]$ and $[\mathrm{S}]$. A good introduction can also be found in [M2]. For multiplier Hopf algebras and algebraic quantum groups, we refer to [VD3, VD5, VD6]. See also [VD4] and [VD-Z1] for related material. The $\mathrm{C}^{*}$-version of algebraic quantum groups has been treated in $[\mathrm{K}-\mathrm{VD}]$ and $[\mathrm{K}]$. For dual pairs of Hopf algebras, we have [VD2] and for dual pairs of multiplier Hopf algebras (more important for this paper), we have [D-VD]. For coactions, we have [VD-Z3].

For actions of Hopf algebras and for smash products, see [M2]. Some standard results about actions of locally compact groups on von Neumann algebras and $\mathrm{C}^{*}$ algebras can be found in $[\mathrm{P}]$ and [VD1].

In general, we work with (associative) algebras over $\mathbb{C}$. Our algebras need not have an identity, but the product is always required to be non-degenerate (as a bilinear map). The fact that our algebras need not have an identity is very important, but it also causes some extra problems. Throughout the paper, we will explain on several occasions what kind of problems occur. We will have a more or less standard way to overcome these. This will be explained in the different situations. If we have an identity (like in the multiplier algebra), we will always denote it by 1 .

The space of all linear functionals on a vector space $A$ (the dual) will always be denoted by $A^{\prime}$. Tensor products are algebraic tensor products (as we are not working in a topological context). We identify $A \otimes \mathbb{C}$ and $\mathbb{C} \otimes A$ with $A$. We use $\iota$ for the identity map. When $\omega$ is a linear functional on $A$, the slice maps $\iota \otimes \omega$ and $\omega \otimes \iota$ are maps from $A \otimes A$ to $A$.

We use $\Delta$ for the comultiplication, both for Hopf algebras and for multiplier Hopf 
algebras. In both cases, we also use $S$ for the antipode and $\epsilon$ for the counit. Ocassionally, we will use $m$ for the multiplication in an algebra $A$ as a linear map from $A \otimes A$ to $A$. Sometimes however, we use $m$ to denote a multiplier.

We will work with the Sweedler summation convention and denote $\Delta(a)$ by $\sum a_{(1)} \otimes$ $a_{(2)}$, not only for usual Hopf algebras, but also for multiplier Hopf algebras. This presents no problem in the first case, but it is somewhat problematic for multiplier Hopf algebras. The use of the Sweedler notation for multiplier Hopf algebras was introduced in [D-VD]. In section 2 of this paper, we will give some more comments about this topic. There, we will also say more about the use of the Sweedler notation in this paper.

Not only when using the Sweedler notation, but also in the case of other summations, we will mostly omit the summation variable. This should be clear in these cases.

Let us finish this introduction with a remark.

It should be clear from the discussion above that the theory of actions and crossed (or smash) products has developed in different fields of mathematics. We have actions of Hopf algebras on the one hand, but also all sorts of actions in the theory of operator algebras, involving different aspects of abstract harmonic analysis. This can also be seen from a view at the list of references. The terminology is not always the same. In operator algebras, one is used to work with actions, covariant systems, representations, covariant representations, crossed products, ... Crossed products and tensor products are usually completed for an appropriate topology. In algebra, one works with modules, module algebras, smash products, ... And of course, there is no completion and tensor products are just algebraic tensor products.

Also the three authors of this paper have different roots. These different backgounds have led to a fruitful cooperation. In operator algebras e.g., one is used to work with algebras without identity. Considering those has led to the notion of multiplier Hopf algebras; in fact, a very natural extension of the notion of Hopf algebras if the assumption is dropped that 'all algebras have an identity'. This has also led to more cases where integrals exist (e.g. the algebraic quantum groups [VD6] and in particular, the discrete quantum groups ([E-R] and [VD4])).

It also had some influence, not only on the terminology used in this paper, but also on the kind of results that are proven.

We have tried to make this paper accessable for readers with these different backgrounds (algebra, analysis, mathematical physics, ...). In fact, we do hope that our theory will eventually lead to a comprehensive theory of actions of locally compact quantum groups, including not only the actions of Hopf algebras and multiplier 
Hopf algebras, but also the different types of actions of locally compact groups and its generalizations.

\section{Multiplier Hopf Algebras}

Multiplier Hopf algebras were introduced in [VD3] in 1994. Since the introduction, certain new results have been obtained. In [VD6] we have given a characterization of the regular multiplier Hopf algebras in terms of the bijectivity of the antipode. In $[\mathrm{D}-\mathrm{VD}]$ we have introduced the use of the Sweedler notation for multiplier Hopf algebras.

In this section we first recall some of the main definitions and results in the theory of multiplier Hopf algebras. But also in this paper, we will obtain some new results (see proposition 2.2 and 2.6 below).

First, recall the definition of a multiplier Hopf algebra (see [VD3] for details).

Let $A$ be an algebra over $\mathbb{C}$, with or without identity, but with a non-degenerate product. The multiplier algebra $M(A)$ can be characterized as the largest algebra with identity in which $A$ sits as a dense (= essential) two-sided ideal. Consider the tensor product $A \otimes A$ of $A$ with itself. This is again an algebra over $\mathbb{C}$ with a non-degenerate product. The imbedding of $A \otimes A$ in the multiplier algebra $M(A \otimes A)$ is factored through the natural imbeddings of $A \otimes A$ in $M(A) \otimes M(A)$ and of $M(A) \otimes M(A)$ in $M(A \otimes A)$.

A comultiplication (or a coproduct) on $A$ is a homomorphism $\Delta: A \rightarrow M(A \otimes A)$ such that $\Delta(a)(1 \otimes b)$ and $(a \otimes 1) \Delta(b)$ are elements of $A \otimes A$ for all $a, b \in A$. It is assumed to be coassociative in the sense that

$$
(a \otimes 1 \otimes 1)(\Delta \otimes \iota)(\Delta(b)(1 \otimes c))=(\iota \otimes \Delta)((a \otimes 1) \Delta(b))(1 \otimes 1 \otimes c)
$$

for all $a, b, c \in A$ (where $\iota$ denotes the identity map).

Then recall the following definition (see [VD3]).

2.1 Definition A pair $(A, \Delta)$ of an algebra $A$ over $\mathbb{C}$ with a non-degenerate product and a comultiplication $\Delta$ on $A$ is called a multiplier Hopf algebra if the linear maps from $A \otimes A$ to itself, defined by

$$
\begin{aligned}
& a \otimes b \rightarrow \Delta(a)(1 \otimes b) \\
& a \otimes b \rightarrow(a \otimes 1) \Delta(b)
\end{aligned}
$$

are bijective.

The motivating example comes from a group. If $G$ is any group, let $A$ be the algebra of complex functions on $G$ with finite support and identify $A \otimes A$ with the 
algebra of complex functions on $G \times G$ with finite support and $M(A \otimes A)$ with the algebra of all complex functions. Then $A$ is made into a multiplier Hopf algebra if we define $\Delta$ by $(\Delta f)(p, q)=f(p q)$ whenever $f \in A$ and $p, q \in G$.

For any multiplier Hopf algebra, we have a counit and an antipode. The counit is the unique linear map $\epsilon: A \rightarrow \mathbb{C}$ satisfying

$$
\begin{aligned}
& (\epsilon \otimes \iota)(\Delta(a)(1 \otimes b))=a b \\
& (\iota \otimes \epsilon)((a \otimes 1) \Delta(b))=a b
\end{aligned}
$$

for all $a, b \in A$. It is a homomorphism. The antipode is the unique linear map $S: A \rightarrow M(A)$ satisfying

$$
\begin{aligned}
& m(S \otimes \iota)(\Delta(a)(1 \otimes b))=\epsilon(a) b \\
& m(\iota \otimes S)((a \otimes 1) \Delta(b))=\epsilon(b) a
\end{aligned}
$$

where $m$ denotes multiplication defined as a linear map from $A \otimes A$ to $A$ and extended to $M(A) \otimes A$ and $A \otimes M(A)$. The antipode is a anti-homomorphism.

In the group example, $\epsilon$ is given by $\epsilon(f)=f(e)$ where $e$ is the identity in $G$ and $S$ is given by $(S(f))(p)=f\left(p^{-1}\right)$ whenever $f \in A$ and $p \in G$.

If $A$ is an algebra with identity, then $M(A)=A$ and $M(A \otimes A)=A \otimes A$ and clearly, when $\Delta$ is a comultiplication on $A$ making it into a multiplier Hopf algebra, then it is actually a Hopf algebra. Conversely, every Hopf algebra (over $\mathbb{C}$ ) is a multiplier Hopf algebra. So, multiplier Hopf algebras are the natural extensions of Hopf algebras to the case where the underlying algebra has no identity.

If $\Delta$ is comultiplication on $A$ such that also $\Delta(a)(b \otimes 1)$ and $(1 \otimes a) \Delta(b)$ are in $A \otimes A$ for all $a, b \in A$, we can consider the opposite comultiplication $\Delta^{\prime}$ obtained from $\Delta$ by composing it with the flip on $A \otimes A$ (extended to $M(A \otimes A)$ ). A multiplier Hopf algebra $(A, \Delta)$ is called regular if also $\left(A, \Delta^{\prime}\right)$ is a multiplier Hopf algebra (i.e. satisfies the conditions in definition 2.1). Regularity is automatic in certain special cases: when $A$ is abelian, when $\Delta$ is coabelian and when $A$ is a *-algebra and $\Delta$ a *homomorphism. The regular multiplier Hopf algebras are precisely those for which the antipode maps $A$ into $A$ and is bijective (see [VD6]). In the ${ }^{*}$-algebra case, when $\Delta$ is a ${ }^{*}$-homomorphism, the antipode satisfies the relation $S\left(a^{*}\right)=S^{-1}(a)^{*}$ for all $a \in A$.

In this paper we will only work with regular multiplier Hopf algebras. Nevertheless, we have the feeling that many results are still valid for multiplier Hopf algebras that are not regular. To obtain such results, one has to be much more careful. For convenience, we have chosen to concentrate first on the regular case.

So, in what follows let $(A, \Delta)$ be a regular multiplier Hopf algebra.

2.2 Proposition Let $a_{1}, a_{2}, \ldots, a_{n}$ be elements in $A$. Then there exist elements $e, f$ in $A$ such that $e a_{i}=a_{i}$ and $a_{i} f=a_{i}$ for all $i$. 
Proof : Take elements $a_{1}, a_{2}, \ldots, a_{n}$ in $A$ and consider the subspace $V$ of $A^{n}$ given by

$$
V=\left\{\left(a a_{1}, a a_{2}, \ldots, a a_{n}\right) \mid a \in A\right\} .
$$

The existence of such an element $e$ follows if $\left(a_{1}, a_{2}, \ldots, a_{n}\right) \in V$. Suppose this is not the case. Then, choose linear functionals $\omega_{1}, \omega_{2}, \ldots, \omega_{n}$ on $A$ such that

$$
\sum \omega_{i}\left(a a_{i}\right)=0
$$

for all $a \in A$ but $\sum \omega_{i}\left(a_{i}\right) \neq 0$. Take any $a \in A$ and write

$$
\Delta(a)\left(1 \otimes a_{i}\right)=\sum_{j} p_{i j} \otimes q_{i j}
$$

with $p_{i j}, q_{i j} \in A$. For all $b \in A$, we get

$$
\sum_{i, j} \omega_{i}\left(b q_{i j}\right) p_{i j}=\sum_{i}\left(\iota \otimes \omega_{i}\right)\left((1 \otimes b) \Delta(a)\left(1 \otimes a_{i}\right)\right)=0
$$

Now, take a basis $\left(e_{k}\right)$ in the (finite-dimensional) subspace $W$ of $A$ spanned by the elements $\left\{S p_{i j} \mid i, j\right\}$ and consider a dual basis, i.e. linear functionals $\left(f_{k}\right)$ such that $f_{k}\left(e_{j}\right)=0$ when $k \neq j$ and $f_{k}\left(e_{k}\right)=1$. Then, for all $x \in W$ we have $\sum_{k} f_{k}(x) e_{k}=x$. Hence

$$
\begin{aligned}
\sum_{i, j} \omega_{i}\left(S\left(p_{i j}\right) q_{i j}\right) & =\sum_{i, j, k} \omega_{i}\left(e_{k} q_{i j}\right) f_{k}\left(S\left(p_{i j}\right)\right) \\
& =\sum_{k} f_{k}\left(S\left(\sum_{i, j} \omega_{i}\left(e_{k} q_{i j}\right) p_{i j}\right)\right)=0
\end{aligned}
$$

Now, for all $i$ we have $\sum_{j} S\left(p_{i j}\right) q_{i j}=\epsilon(a) a_{i}$ by the property of the antipode. Hence $\sum \omega_{i}\left(a_{i}\right) \epsilon(a)=0$. So, if we choose $a$ such that $\epsilon(a) \neq 0$ we would get $\sum \omega_{i}\left(a_{i}\right)=0$ and this leads to a contradiction.

This proves the existence of $e$. Similarly, or by applying the antipode, we get the existence of $f$.

As we can see in the proof, we do not need the fact that $S$ is bijective to prove that $e$ exists, but we have used that $S(A) \subseteq A$. So, it is not clear if the previous result remains true for any multiplier Hopf algebra. (In fact we even don't know of examples of multiplier Hopf algebras for which $S(A)$ does not lie in $A$ !).

The property of $A$ that we proved in proposition 2.2 has some simple but nice (and important) consequences.

First remark of course that the property is trivial for algebras with an identity. Perhaps the main consequence is the justification of the use of the Sweedler notation, certainly for this paper. The Sweedler notation for multiplier Hopf algebras was 
first used in $[\mathrm{D}-\mathrm{VD}]$. Using the property above, we can justify the Sweedler notation in this context even more.

We would like to use a formal expression for $\Delta(a)$ when $a \in A$. The problem is that $\Delta(a)$ is not in $A \otimes A$ in general. We know however that $\Delta(a)(1 \otimes b) \in A \otimes A$ for all $a, b \in A$. We have proposed in [D-VD] to write $\sum_{(a)} a_{(1)} \otimes a_{(2)} b$ for this. Now, we know that there is an element $e \in A$ such that $b=e b$ and we can think of $\sum_{(a)} a_{(1)} \otimes a_{(2)}$ to stand for $\Delta(a)(1 \otimes e)$. Of course, this is still dependent on $b$. But we know that for several elements $b$, we can use the same $e$.

When using Sweedler's notation for regular multiplier Hopf algebras, one always has to make sure that at most one factor $a_{(k)}$ is not covered by an element of $A$. Expressions like $\sum a_{(1)} \otimes b a_{(2)}$ and $\sum a_{(1)} \otimes b a_{(2)} \otimes a_{(3)} c$ are allowed. For this last expression take e.g. elements $e, f$ in $A$ such that $b f=b$ and $e c=c$ and use $\sum a_{(1)} \otimes f a_{(2)} \otimes a_{(3)} e$ for $(1 \otimes f \otimes 1)(\Delta \otimes \iota)(\Delta(a)(1 \otimes e))$ which is well-defined in $A \otimes A \otimes A$.

Nevertheless, the Sweedler notation should be used with some care and it is good to have in mind what the expression stands for. One situation where one has to be particularly careful is when using the Sweedler notation in defining maps (because of the ambiguity). If any doubt, it is always possible to avoid it. On the other hand, the Sweedler notation is very convenient and makes formulas much more transparant. It has been our choice to make use of it in this paper, also if, at first sight, it is somewhat dangerous. In any case, the reader can easily translate the formulas to a form which does not contain the Sweedler notation.

Let us now turn our attention to a second consequence of proposition 2.2. First recall that for any $a \in A$ and $\omega \in A^{\prime}$ we can define elements $(\omega \otimes \iota) \Delta(a)$ and $(\iota \otimes \omega) \Delta(a)$ in $M(A)$. Now we can look at the subspace of $A^{\prime}$ of elements $\omega$ such that $(\omega \otimes \iota) \Delta(a)$ and $(\iota \otimes \omega) \Delta(a)$ are in $A$ for all $a \in A$. We know that elements of the form $\omega(\cdot b)$ and $\omega(b \cdot)$ where $\omega \in A^{\prime}$ and $b \in A$ have this property. Now it follows from the proposition that these functionals also form a subspace of $A^{\prime}$. Indeed, take e.g. $\omega, \psi \in A^{\prime}$ and $b, c \in A$. Choose $e \in A$ so that $e b=b$ and $e c=c$. Define $\rho \in A^{\prime}$ by $\rho(a)=\omega(a b)+\psi(a c)$. Then $\rho=\rho(\cdot e)$ so that also $\rho$ has this form.

It also follows from this proposition that elements of the form $\rho=\omega(\cdot b)$ have unique extensions to $M(A)$ satisfying $\rho(x)=\omega(x b)$ for all $x \in M(A)$. Indeed, if again $\omega, \psi \in A^{\prime}$ and $b, c \in A$ and if now $\omega(a b)=\psi(a c)$ for all $a \in A$, then again take $e$ such that $e b=b$ and $e c=c$ and we find for all $x \in M(A)$ that $\omega(x b)=\omega(x e b)=\psi(x e c)=\psi(x c)$.

These two other consequences are not so important for this paper. They were used e.g. in [VD-Z1].

Next, let us recall the notion of an integral. 
2.3 Definition Let $(A, \Delta)$ be a multiplier Hopf algebra. A linear functional $\varphi$ is called a left integral if it is non-zero and if $(\iota \otimes \varphi) \Delta(a)=\varphi(a) 1$ for all $a \in A$. A non-zero linear functional $\psi$ is called a right integral if $(\psi \otimes \iota) \Delta(a)=\psi(a) 1$ for all $a \in A$.

It has been shown in [VD6] that integrals are unique (up to a scalar of course) for regular multiplier Hopf algebras. They are also faithful (i.e. if $\varphi(a \cdot)=0$ then $a=0$ and if $\varphi(\cdot a)=0$, then $a=0)$. They satisfy the so-called weak K.M.S. property: there is an automorphism $\sigma$ of $A$ such that $\varphi(a b)=\varphi(b \sigma(a))$ for all $a, b \in A$. It is also clear that the antipode converts a left integral to a right one and the other way around.

We come to the following definition.

2.4 Definition An algebraic quantum group is a regular multiplier Hopf algebra with integrals.

Algebraic quantum groups have been studied in [VD6]. The main result is duality:

2.5 Theorem Let $(A, \Delta)$ be an algebraic quantum group. Let $\varphi$ be a left integral. Set $\hat{A}=\{\varphi(\cdot a) \mid a \in A\}$. Considering $\hat{A}$ as a subspace of $A^{\prime}$, the dual of the coproduct and the product of $A$ give a product and a coproduct $\hat{\Delta}$ on $\hat{A}$ such that $(\hat{A}, \hat{\Delta})$ is again an algebraic quantum group.

We call $(\hat{A}, \hat{\Delta})$ the dual of $(A, \Delta)$. It is also shown in [VD6] that the dual of $(\hat{A}, \hat{\Delta})$ is canonically isomorphic with $(A, \Delta)$.

For algebraic quantum groups, we can push the result of 2.2 a little further:

2.6 Proposition Let $(A, \Delta)$ be an algebraic quantum group. Given elements $\left(a_{1}, \ldots, a_{n}\right)$ in $A$, there exists an element $e \in A$ such that $a_{i} e=e a_{i}=a_{i}$ for all $i$.

Proof : Let $\omega_{i}, \rho_{i}$ be linear functionals so that

$$
\sum \omega_{i}\left(b a_{i}\right)+\sum \rho_{i}\left(a_{i} b\right)=0
$$

for all $b \in A$. Then, as in the proof of 2.2 , we have to show now that

$$
\sum \omega_{i}\left(a_{i}\right)+\sum \rho_{i}\left(a_{i}\right)=0
$$

But, when $\sum \omega_{i}\left(b a_{i}\right)+\sum \rho_{i}\left(a_{i} b\right)=0$ for all $b \in A$, then also

$$
\sum\left(\omega_{i} \otimes \iota\right)\left(\left(\Delta(b)\left(a_{i} \otimes 1\right)\right)+\sum\left(\rho_{i} \otimes \iota\right)\left(\left(a_{i} \otimes 1\right) \Delta(b)\right)=0\right.
$$

for all $b \in A$. If we apply a left integral $\varphi$ we get $\varphi(b)\left(\sum \omega_{i}\left(a_{i}\right)+\sum \rho_{i}\left(a_{i}\right)\right)=0$. So, just take any $b$ so that $\varphi(b) \neq 0$. 
Observe that the proof here is simpler than in 2.2 and that the result is slightly stronger. We have to mention that 2.6 first has been proved by J. Kustermans, but his proof was more complicated.

Again, this stronger result makes the use of the Sweedler notation for algebraic quantum groups even more acceptable. Consider e.g. an element $a$ and a finite number of elements $a_{i}, b_{i}, c_{i}, d_{i}$. Then we can choose one element $e$ so that $e a_{i}=a_{i}$ for all $i$ and

$$
\begin{aligned}
\Delta(a)\left(b_{i} \otimes 1\right) & =\left(\Delta(a)\left(b_{i} \otimes 1\right)\right)(1 \otimes e) \\
& =(\Delta(a)(1 \otimes e))\left(b_{i} \otimes 1\right) \\
\left(1 \otimes c_{i}\right) \Delta(a) & =\left(\left(1 \otimes c_{i}\right) \Delta(a)\right)(1 \otimes e) \\
& =\left(1 \otimes c_{i}\right)(\Delta(a)(1 \otimes e)) \\
\left(d_{i} \otimes 1\right) \Delta(a) & =\left(\left(d_{i} \otimes 1\right) \Delta(a)\right)(1 \otimes e) \\
& =\left(d_{i} \otimes 1\right)(\Delta(a)(1 \otimes e))
\end{aligned}
$$

for all $i$. So, if we use $\sum a_{(1)} \otimes a_{(2)}$ for $\Delta(a)(1 \otimes e)$, we can write

$$
\begin{aligned}
& \sum a_{(1)} \otimes a_{(2)} a_{i}=\Delta(a)\left(1 \otimes a_{i}\right) \\
& \sum a_{(1)} b_{i} \otimes a_{(2)}=\Delta(a)\left(b_{i} \otimes 1\right) \\
& \sum a_{(1)} \otimes c_{i} a_{(2)}=\left(1 \otimes c_{i}\right) \Delta(a) \\
& \sum d_{i} a_{(1)} \otimes a_{(2)}=\left(d_{i} \otimes 1\right) \Delta(a)
\end{aligned}
$$

for all $i$.

In connection with the other remark we made in the general case, we can mention that for algebraic quantum groups, the set of functionals $\omega \in A^{\prime}$ such that $(\omega \otimes$ $\iota) \Delta(a)$ and $(\iota \otimes \omega) \Delta(a)$ are in $A$ for all $a \in A$ precisely coincides with $M(\hat{A})$ (see $[\mathrm{K}])$.

Algebraic quantum groups will play an important rôle in this paper. In fact, the main duality result in section 7 will only be proven for an algebraic quantum group.

For completeness, we now recall also the notions of algebraic quantum groups of discrete and compact type. These two special cases will not play a special rôle here. Nevertheless, they are the best known types of algebraic quantum groups and so, it is good to recall their definitions.

First recall the definition of a cointegral.

2.7 Definition Let $(A, \Delta)$ be a multiplier Hopf algebra. A non-zero element $h \in A$ is called a left cointegral if $a h=\epsilon(a) h$ for all $a \in A$. Similarly, a non-zero element $k$ in $A$ is called a right cointegral if $k a=\epsilon(a) k$ for all $a \in A$. 
It has been shown in [VD-Z1] that also cointegrals are unique (up to a scalar) for regular multiplier Hopf algebras. They are faithful in the sense that $(\omega \otimes \iota) \Delta(h)=0$ and $\omega \in A^{\prime}$ implies $\omega=0$ and $(\iota \otimes \omega) \Delta(h)=0$ implies $\omega=0$. Again the antipode will convert a left cointegral into a right one and a right one into a left one.

Regular multiplier Hopf algebras with cointegrals have been studied in [VD-Z1]. There it is show that integrals exist in this case. We also have obtained some necessary and sufficient conditions on the underlying algebra $A$ for $(A, \Delta)$ to have cointegrals. We have the following definition and result.

2.8 Definition A regular multiplier Hopf algebra with cointegrals is called a multiplier Hopf algebra of discrete type (or an algebraic quantum group of discrete type). A Hopf algebra with integrals is called (an algebraic quantum group) of compact type.

It is shown in [VD6] that the dual of a discrete type is of compact type and of course that the dual of a compact type is of discrete type. Because for the discrete type, we have integrals and cointegrals, we get a class of multiplier Hopf algebras that shares most properties with the finite-dimensional Hopf algebras.

In the discrete type case, we have shown in [VD-Z1] that the elements $e$ obtained in proposition 2.6 can be chosen to be idempotent. The existence of these local units is obvious in the case of a discrete group $G$. Then, $A$ is the algebra $K(G)$ of complex functions with finite support on $G$ (and pointwise operations). The local units are the functions that are 1 on a finite subset of $G$ and 0 outside this subset. Similarly in the case of a discrete quantum group in the sense of [E-R] and [VD4]. In this case, the underlying algebra is a direct sum of matrix algebras and the local units are the sums of finitely many identities of these components. For another example, see [VD6] and [VD-Z1].

In the discrete type case, we also have that $M(\hat{A})=\hat{A}$ as $\hat{A}$ has an identity. So, the set of functionals $\omega \in A^{\prime}$ satisfying $(\omega \otimes \iota) \Delta(a) \in A$ and $(\iota \otimes \omega) \Delta(a) \in A$ for all $a \in A$ are precisely those in $\hat{A}$.

\section{Multiplier Hopf Algebra Modules}

In this section we will study some basic properties of left $A$-modules where $A$ is the underlying algebra of a multiplier Hopf algebra. In particular, in what follows, $A$ will be an algebra over $\mathbb{C}$, with or without an identity but with a non-degenerate product and satisfying property 2.2 of the previous section.

We consider a left $A$-module $R$. So, $R$ is a vector space over $\mathbb{C}$ and we have a bilinear map $(a, x) \in A \times R \rightarrow a x \in R$ satisfying $\left(a a^{\prime}\right) x=a\left(a^{\prime} x\right)$ for all $a, a^{\prime} \in A$ and $x \in R$. 
Left $A$-modules where already considered in $[\mathrm{D}-\mathrm{VD}]$. Here, we will use a slightly different terminology.

If the algebra $A$ has an identity, it is normal to assume that $1 x=x$ for all $x \in R$. This means that the module is unital. In our context, it seems to be most natural to extend this notion in the following way:

3.1 Definition Let $R$ be a left $A$-module. We call $R$ unital if $A R=R$.

It is clear that this notion coincides with the condition $1 x=x$ for all $x$ when $A$ has an identity.

We also have the following easy consequence.

3.2 Proposition Let $R$ be a unital left $A$-module. If $x \in R$ and $a x=0$ for all $a \in A$, then $x=0$.

Proof : So, let $x \in R$ and assume that $a x=0$ for all $a \in A$. By assumption, we can write $x=\sum a_{i} x_{i}$ where $a_{i} \in A$ and $x_{i} \in R$ for all $i$. Choose $e \in A$ such that $e a_{i}=a_{i}$ for all $i$. Then $e x=x$. But $e x=0$ and hence $x=0$.

We say that a unital $A$-module is non-degenerate.

Remark that in [D-VD] we call a module non-degenerate if $\mathrm{R}$ is unital and satisfies the property in proposition 3.2. This ambiguity has to do with the notion of a non-degenerate ${ }^{*}$-representation $\pi$ of (say) a $\mathrm{C}^{*}$-algebra $B$ on a Hilbert space $\mathcal{H}$. In that case, the density of $\pi(B) \mathcal{H}$ in $\mathcal{H}$ is equivalent with the non-degeneracy condition $\pi(B) \xi=0$ implies $\xi=0$ (which is essentially the condition in 3.2). See $[\mathrm{P}]$.

This observation might suggest that the converse of proposition 3.2 is true. This is not the case. Consider $A^{\prime}$ as a left $A$ module by $a \omega=\omega(\cdot a)$. This module is non-degenerate because if $\omega(\cdot a)=0$ for all $a$, then $\omega=0$. On the other hand, in general, we do not have that $A^{\prime}=A A^{\prime}$.

So we see that in this purely algebraic context, the notion of non-degeneracy (as formulated in proposition 3.2) is weaker than the notion unital. This is different in the topological context when dealing with *-algebras.

We believe that many results in this paper can still be obtained under the weaker condition. In some cases however, it may be necessary to have the stronger one. Also, for other reasons, it seems very natural to assume the condition $A R=R$. We have e.g. that $A A=A$ and conditions like $\Delta(A)(1 \otimes A)=A \otimes A$. Also, when we consider a homomorphism from one algebra into the multiplier algebra of another one, we will require a similar condition (see definition 4.12 in the next section). So, because it seems very natural and for convenience, we will assume throughout the paper that we are dealing with unital modules. There are a few exceptions but we will indicate these very clearly. 
Remark that for a unital $A$-module $R$, for any $x \in R$ there is an $e \in A$ such that $e x=x$. This was used in the proof of the proposition. In fact, using the same argument, we have for all $x_{1}, x_{2}, \ldots, x_{n} \in R$ an element $e$ such that $e x_{i}=x_{i}$ for all $i$. This is important for working with the Sweedler notation: it means that elements in a unital left $A$-module will cover elements $a_{(k)}$.

Next we show that unital $A$-modules can be extended to modules over $M(A)$. This result can already be found in [D-VD].

3.3 Proposition Let $R$ be a unital left $A$-module. Then, there is a unique extension to a left $M(A)$-module. We have $1 x=x$ for all $x \in R$ where now $1 \in M(A)$.

Proof : If $M(A)$ also acts on $R$ and if this action extends the action of $A$, we must have $m(a x)=(m a) x$ for all $x \in R, a \in A$ and $m \in M(A)$. Because $R$ is unital, this relation completely determines the action of $M(A)$ and we must have $1 x=x$ for all $x$. On the other hand, we can use this formula to define the action of $M(A)$. To prove that this is well-defined, assume that $a_{i} \in A$ and $x_{i} \in R$ and that $\sum a_{i} x_{i}=0$. Choose $e \in A$ so that $e a_{i}=a_{i}$ for all $i$. For any $m \in M(A)$ we have

$$
\sum\left(m a_{i}\right) x_{i}=\sum(m e)\left(a_{i} x_{i}\right)=(m e) \sum a_{i} x_{i}=0 .
$$

It follows that we can define the action of $M(A)$ by $m(a x)=(m a) x$ whenever $a \in A, x \in R$ and $m \in M(A)$.

Suppose that we did not have an algebra with local units (as in propositioin 2.2). Then also proposition 3.3 might fail. However, if $R$ is both unital and nondegenerate, we still have 3.3. Indeed, observe that, if $\sum a_{i} x_{i}=0$, then for all $b \in A$ and $m \in M(A)$ we get

$$
b\left(\sum\left(m a_{i}\right) x_{i}\right)=\sum\left(b m a_{i}\right) x_{i}=(b m) \sum a_{i} x_{i}=0 .
$$

Because the module is non-degenerate we obtain $\sum\left(m a_{i}\right) x_{i}=0$ (see again [D-VD]). We will come back to this problem in section 5 where we will also consider modules over the smash product (which need not to have such local units).

Now, let $(A, \Delta)$ be a regular multiplier Hopf algebra. Then we can use $\Delta$ to define tensor products of unital left $A$-modules. Indeed, suppose that $R$ and $T$ are unital left $A$-modules. Then we can make $R \otimes T$ into a left $(A \otimes A)$-module by $(a \otimes b)(x \otimes y)=a x \otimes b y$. It is clear that also this module will be unitial. Hence, we can use proposition 3.3 to extend it to $M(A \otimes A)$. Then it is possible to use $\Delta$ to get an action of $A$ on $R \otimes T$ given by

$$
a(x \otimes y)=\Delta(a)(x \otimes y)
$$


Now, because $\Delta(A)(A \otimes A)=A \otimes A$ we get

$$
\begin{aligned}
\Delta(A)(R \otimes T) & =\Delta(A)(A \otimes A)(R \otimes T) \\
& =(A \otimes A)(R \otimes T) \\
& =R \otimes T
\end{aligned}
$$

so that also the action of $A$ on $R \otimes T$ is a unital action.

In fact, we get the following result.

3.4 Proposition Let $A$ be a regular multiplier Hopf algebra. Denote by $\mathcal{M}$ the category of unital left $A$-modules and morphisms. Then $\mathcal{M}$ is a monoidal category with unit (for the product structure defined above and with the diagonal $A$-module structure).

The unit is of course given by $\mathbb{C}$. The module structure in this case is $a \lambda=\epsilon(a) \lambda$ whenever $a \in A$ and $\lambda \in \mathbb{C}$. It is clear that this is a unit because $\Delta(a)(\lambda \otimes x)=$ $\lambda(a x)=a(\lambda x)$ for all $a \in A, x \in R$ and $\lambda \in \mathbb{C}$.

In prinicple, when $R$ is a unital left $A$-module, we can also define the fixed points in $R$ as the elements $x \in R$ that satisfy $a x=\epsilon(a) x$ for all $a \in A$. This notion is a little too restrictive. Think e.g. of the algebra of complex functions with finite support on a group $G$ and let it act on itself by left convolution. This will give a unital action of the group Hopf algebra $\mathbb{C}$. There will be no fixed points. Only constant functions would be fixed points, but these are not in our algebra. So, in fact, it is more natural to look at fixed points in the multiplier algebra of $R$. But to do this, one first has to extend the action of $A$ to this multiplier algebra. This will be done in section 4 .

For the module actions, we will use different notations. Here, we have used $a x$ for the action of $a$ on $x$. In some papers, $a . x$ is used instead. Sometimes, certainly when more different types of actions are involved, we will also use $a \triangleright x$.

\section{Module Algebras over Multiplier Hopf Algebras}

Again, let $(A, \Delta)$ be a regular multiplier Hopf algebra. Now assume that $R$ is an algebra over $\mathbb{C}$, with or without identity but with non-degenerate product. Assume that $R$ is a unital left $A$-module, cf. definition 3.1 .

The main definition in this section is the following.

4.1 Definition We say that $R$ is a left $A$-module algebra if

$$
a\left(x x^{\prime}\right)=\sum\left(a_{(1)} x\right)\left(a_{(2)} x^{\prime}\right)
$$


for all $a \in A$ and $x, x^{\prime} \in R$.

Let us first discuss the right hand side of the above equality. We have seen in the previous section that the elements $x$ and $x^{\prime}$ can be used to cover $a_{(1)}$ and $a_{(2)}$. But it is also possible to view this expression as $m\left(\Delta(a)\left(x \otimes x^{\prime}\right)\right)$ where now $m$ denotes multiplication in $R$. Indeed, we have seen in proposition 3.3 that this makes sense because $R$ is unital. Then, the condition in 4.1 can be rewritten as $m \Delta(a)=a m$, saying that $m$ is an $A$-module map.

When $R$ is a left $A$-module algebra, we will also say that the multiplier Hopf algebra $A$ acts on $R$. This last formulation is closer to the notion of an action of a group (which in fact is the original situation). See example 4.3 below.

Before we give some examples however, let us first prove some immediate consequences of the definition. In fact, in the following lemma, we give some formulas that are essentially equivalent with the formula in the definition above.

4.2 Lemma For any $a \in A$ and $x, x^{\prime} \in R$ we have

$$
\begin{aligned}
& (a x) x^{\prime}=\sum a_{(1)}\left(x\left(S\left(a_{(2)}\right) x^{\prime}\right)\right) \\
& x\left(a x^{\prime}\right)=\sum a_{(2)}\left(\left(S^{-1}\left(a_{(1)}\right) x\right) x^{\prime}\right) .
\end{aligned}
$$

Proof : We have

$$
\begin{aligned}
\sum a_{(1)}\left(x\left(S\left(a_{(2)}\right) x^{\prime}\right)\right) & =\sum\left(a_{(1)} x\right)\left(a_{(2)} S\left(a_{(3)}\right) x^{\prime}\right) \\
& =\sum \epsilon\left(a_{(2)}\right)\left(a_{(1)} x\right) x^{\prime}=(a x) x^{\prime}
\end{aligned}
$$

and similarly for the other formula.

Remark that $x^{\prime}$ covers $a_{(2)}$ in the first formula and that $a_{(1)}$ is covered by $x$ in the second formula of the lemma.

We will use these formulas at several places in the paper. In particular, we will use them later in this section to extend the action of $A$ on $R$ to $M(R)$.

Let us now consider some examples. We start with the natural one. There is nothing new about this example because we have an example of an action of a Hopf algebra. But it serves as a motivation, also for actions of multiplier Hopf algebras.

4.3 Example Let $G$ be a group and let $A$ be the group algebra $\mathbb{C} G$. Denote the imbedding of $G$ in $A$ by $p \rightarrow \lambda_{p}$ so that $A$ is spanned by the elements $\left\{\lambda_{p} \mid p \in G\right\}$ and that $\Delta$ is given by $\Delta\left(\lambda_{p}\right)=\lambda_{p} \otimes \lambda_{p}$ for all $p \in G$. Now, let $\alpha$ be an action of $G$ on an algebra $R$ by means of automorphisms of $R$. We associate an action of $A$ on $R$ by $\lambda_{p} x=\alpha_{p}(x)$ whenever $p \in G$ and $x \in R$. Because $\alpha$ is an action of $G$, we have that $R$ is an $A$-module. Since 
also $\alpha_{e}(x)=x$ for all $x \in R$ when $e$ is the identity in $G$, we have a unital $A$-module. Finally, because $\alpha_{p}$ is an automorphism, we get

$$
\begin{aligned}
\lambda_{p}\left(x x^{\prime}\right) & =\alpha_{p}\left(x x^{\prime}\right)=\alpha_{p}(x) \alpha_{p}\left(x^{\prime}\right) \\
& =\left(\lambda_{p} x\right)\left(\lambda_{p} x^{\prime}\right) .
\end{aligned}
$$

So, $R$ is a $A$-module algebra.

The next example is (in general) not a Hopf algebra example.

4.4 Example Let $G$ be group and now let $A$ be the algebra of complex functions with finite support in $G$. Let $\Delta$ be defined by $(\Delta f)(p, q)=f(p q)$ for all $p, q \in G$ and $f \in A$. Now denote by $\delta_{p}$ the function that is 1 on $p$ and 0 everywhere else. Suppose that we have an action of $A$ on an algebra $R$. Denote $R_{p}=\delta_{p} R$. It is clear that $\delta_{p}$ acts as a projection map from $R$ to the space $R_{p}$. Moreover, if $R$ is unital, we will get that $R$ can be identified with the direct sum of these subspaces $R_{p}$ with $p \in G$. Because

$$
\Delta\left(\delta_{p}\right)=\sum_{p=q r} \delta_{q} \otimes \delta_{r}
$$

we have that

$$
\delta_{p}\left(x x^{\prime}\right)=\sum_{p=q r}\left(\delta_{q} x\right)\left(\delta_{r} x^{\prime}\right)
$$

In particular, if $x \in R_{q}$ and $x^{\prime} \in R_{r}$ we get $x x^{\prime} \in R_{q r}$. So $R$ is a $G$-graded algebra. In fact, also conversely, if $R$ is a $G$-graded algebra, with grading $R=\oplus_{p \in G} R_{p}$, then $A$ acts on $R$ if we let $\delta_{p} x=x$ when $x \in R_{p}$ and $\delta_{p} x=0$ when $x \in R_{q}$ and $q \neq p$.

Let us now consider the adjoint action. It will play a fundamental rôle in what follows.

4.5 Proposition Let $A$ act on itself by the map $a \otimes x \rightarrow \sum a_{(1)} x S\left(a_{(2)}\right)$ from $A \otimes A$ to $A$. This action makes $A$ into an $A$-module algebra.

Proof : First observe that the above expression makes sense. Also, compare this formula with the first one in lemma 4.2. Because for all $a, b, x \in A$ we get

$$
\begin{aligned}
\sum(a b)_{(1)} x S\left((a b)_{(2)}\right) & =\sum a_{(1)} b_{(1)} x S\left(a_{(2)} b_{(2)}\right) \\
& =\sum a_{(1)} b_{(1)} x S\left(b_{(2)}\right) S\left(a_{(2)}\right),
\end{aligned}
$$

we clearly have a left $A$-module structure. To see that we have a unital module, observe that the surjectivity of the map

$$
a \otimes x \rightarrow \Delta(a)(x \otimes 1)
$$


will give that $p S(q)$ is in the range of this module map for all $p, q \in A$ and so is $A$. Finally, for all $a, x, x^{\prime} \in A$, we also get

$$
\begin{aligned}
\sum\left(a_{(1)} x S\left(a_{(2)}\right)\right)\left(a_{(3)} x^{\prime} S\left(a_{(4)}\right)\right) & =\sum \epsilon\left(a_{(2)}\right) a_{(1)} x x^{\prime} S\left(a_{(3)}\right) \\
& =\sum a_{(1)} x x^{\prime} S\left(a_{(2)}\right)
\end{aligned}
$$

so that we have an $A$-module algebra structure.

Of course, if we consider the adjoint action of proposition 4.5 for the group algebra (example 4.3), we get the adjoint action of $G$ on $\mathbb{C} G$ given by $\alpha_{p}(x)=\lambda_{p} x \lambda_{p}^{-1}$.

Also the following example is closely related with the adjoint action.

4.6 Example Start with a left $A$-module $R$. Let $L$ be the vector space of linear maps from $R$ to $R$. Then $L$ is an algebra with identity (the product is the composition of maps). The action of $A$ yields an action on $L$ given by the following formula. If $\lambda \in L$, let $a \lambda$ be the linear map on $R$ defined by

$$
(a \lambda) x=\sum a_{(1)} \lambda\left(S\left(a_{(2)}\right) x\right) .
$$

Remark that $x$ will cover $a_{(2)}$. Also here, compare this formula with the first formula in lemma 4.2. Similarly as in example 4.5, we have made $L$ into an $A$-module algebra.

Now we want to extend the action of $A$ to the multiplier algebra $M(R)$. Since elements in the multiplier algebra $M(R)$ yield linear maps from $R$ to $R$, we can use the formula in example 4.6 to extend the action of $A$ from $R$ to $M(R)$. This is done in the following proposition. Remark that again, we are motivated by the formulas in lemma 4.2 .

4.7 Proposition We can define $a m \in M(R)$ for all $a \in A$ and $m \in M(R)$ by

$$
\begin{aligned}
& (a m) x=\sum a_{(1)}\left(m\left(S\left(a_{(2)}\right) x\right)\right) \\
& x(a m)=\sum a_{(2)}\left(\left(S^{-1}\left(a_{(1)}\right) x\right) m\right)
\end{aligned}
$$

whenever $x \in R$.

Proof : We will check the relation $\left(x^{\prime}(a m)\right) x^{\prime \prime}=x^{\prime}\left((a m) x^{\prime \prime}\right)$ with the expressions in the formulation of the proposition. For the left hand side, we get

$$
\left(x^{\prime}(a m)\right) x^{\prime \prime}=\sum\left(a_{(2)}\left(\left(S^{-1}\left(a_{(1)}\right) x^{\prime}\right) m\right)\right) x^{\prime \prime} .
$$

For the right hand side we have

$$
\begin{aligned}
x^{\prime}\left((a m) x^{\prime \prime}\right) & =\sum x^{\prime}\left(a_{(1)}\left(m\left(S\left(a_{(2)}\right) x^{\prime \prime}\right)\right)\right) \\
& =\sum\left(a_{(2)} S^{-1}\left(a_{(1)}\right) x^{\prime}\right)\left(a_{(3)}\left(m\left(S\left(a_{(4)}\right) x^{\prime \prime}\right)\right)\right) \\
& =\sum a_{(2)}\left(\left(S^{-1}\left(a_{(1)}\right) x^{\prime}\right)\left(m\left(S\left(a_{(3)}\right) x^{\prime \prime}\right)\right)\right) \\
& =\sum\left(a_{(2)}\left(\left(S^{-1}\left(a_{(1)}\right) x^{\prime}\right) m\right)\right)\left(a_{(3)} S\left(a_{(4)}\right) x^{\prime \prime}\right) \\
& =\sum\left(a_{(2)}\left(\left(S^{-1}\left(a_{(1)}\right) x^{\prime}\right) m\right)\right) x^{\prime \prime}
\end{aligned}
$$


And we find the same.

Remark that in all the formulas, appearing in the proof above, enough elements $a_{(k)}$ are covered. In particular, in the defining relations e.g. $a_{(2)}$ is covered by $x$ in the first one and $a_{(1)}$ by $x$ in the second one.

We have some obvious results on this extended action.

4.8 Proposition We have $\left(a a^{\prime}\right) m=a\left(a^{\prime} m\right)$ for all $a, a^{\prime} \in A$ and $m \in M(R)$. Also $a 1=\epsilon(a) 1$.

Proof : Take $a, a^{\prime} \in A$ and $m \in M(R)$. For all $x \in R$ we get

$$
\begin{aligned}
\left(\left(a a^{\prime}\right) m\right) x & =\sum\left(a a^{\prime}\right)_{(1)}\left(m\left(S\left(\left(a a^{\prime}\right)_{(2)}\right) x\right)\right) \\
& =\sum a_{(1)} a_{(1)}^{\prime}\left(m\left(S\left(a_{(2)}^{\prime}\right) S\left(a_{(2)}\right) x\right)\right) \\
& =\sum a_{(1)}\left(\left(a^{\prime} m\right)\left(S\left(a_{(2)}\right) x\right)\right) \\
& =\left(a\left(a^{\prime} m\right)\right) x .
\end{aligned}
$$

This proves the first statement.

To prove the second statement, take $a \in A$ and $x \in R$. Then

$$
(a 1) x=\sum a_{(1)}\left(S\left(a_{(2)}\right) x\right)=\sum\left(a_{(1)} S\left(a_{(2)}\right) x=\epsilon(a) x .\right.
$$

Hence $a 1=\epsilon(a) 1$.

So, we obtain that $M(R)$ is a left $A$-module. One might guess that still $M(R)$ is a left $A$-module algebra. But there is a problem. One can no longer expect that $M(R)$ is a unital left $A$-module. In general, the condition $A M(R)=M(R)$ will be much too strong. So, we can not give a meaning to the formula

$$
a\left(m m^{\prime}\right)=\sum\left(a_{(1)} m\right)\left(a_{(2)} m^{\prime}\right)
$$

for all $a \in A$ and $m, m^{\prime} \in M(R)$ in the same way as we $\operatorname{did}$ for $R$.

Fortunately, we do have that the action of $A$ on $M(R)$ is still non-degenerate:

4.9 Proposition If $m \in M(R)$ and $a m=0$ for all $a$, then $m=0$.

Proof : For all $a \in A$ and $x \in R$, we have

$$
\begin{aligned}
m(S(a) x) & =\sum S\left(a_{(1)}\right) a_{(2)}\left(m\left(S\left(a_{(3)}\right) x\right)\right. \\
& =\sum S\left(a_{(1)}\right)\left(\left(a_{(2)} m\right) x\right)=0 .
\end{aligned}
$$

As this is true for all $a \in A$ and $x \in R$, and because $R$ is unital, we have $m=0$. 
Now, we are ready to study the fixed points.

We have already mentioned in section 3 that fixed points can be defined in $R$ as the elements $x$ satisfying $a x=\epsilon(a) x$ for all $a \in A$. They obviously form a subalgebra of $R$. But the set of fixed points in $R$ may be too small in general.

It makes more sense to look at fixed points in the multiplier algebra $M(R)$. This is possible since we have extended the action of $A$ to $M(R)$. This gives the following definition.

4.10 Definition A fixed point in $M(R)$ is an element $m$ satisfying $a m=\epsilon(a) m$ for all $a \in A$.

It is not so hard to prove the following property.

4.11 Proposition If $m$ is a fixed point in $M(R)$, then

$$
\begin{aligned}
& a(m x)=m(a x) \\
& a(x m)=(a x) m
\end{aligned}
$$

for all $a \in A$ and $x \in R$.

Proof : Because $m$ is a fixed point, we get for all $a, a^{\prime} \in A$ and $x \in R$

$$
\begin{aligned}
a^{\prime} S(a)(m x) & =\sum a^{\prime} S\left(a_{(1)}\right)\left(a_{(2)} m\right) x \\
& =\sum a^{\prime} S\left(a_{(1)}\right) a_{(2)}\left(m\left(S\left(a_{(3)}\right) x\right)\right) \\
& =a^{\prime} m(S(a) x) .
\end{aligned}
$$

We use $a^{\prime}$ for covering purposes. Now we can cancel $a^{\prime}$ and replace $S(a)$ by $a$ to get $a(m x)=m(a x)$ for all $a \in A$ and $x \in R$. This proves the first formula. Similarly

$$
\begin{aligned}
a^{\prime} S^{-1}(a)(x m) & =\sum a^{\prime} S^{-1}\left(a_{(2)}\right)\left(x\left(a_{(1)} m\right)\right) \\
& =\sum a^{\prime} S^{-1}\left(a_{(3)}\right) a_{(2)}\left(\left(S^{-1}\left(a_{(1)}\right) x\right) m\right) \\
& =a^{\prime}\left(S^{-1}(a) x\right) m
\end{aligned}
$$

and we get the second formula.

This means that multiplication with $m$, both from the left and from the right, commutes with the action of $A$ on $R$. It is not so hard to see that these facts imply that $m$ is a fixed point. So, we could also use this property to define fixed points in $M(R)$ and we do not really need the extension of the action to introduce this notion. It also follows that the fixed points in $M(R)$ form actually a subalgebra. This is not completely obvious from the way we defined it as we do not have that $M(R)$ is an $A$-module algebra. 
We can also look for fixed points in the algebra $L$ of linear maps from $R$ to $R$ for the action considered in example 4.6. So, a linear map $\lambda$ will be a fixed point if

$$
\sum a_{(1)} \lambda\left(S\left(a_{(2)}\right) x\right)=\epsilon(a) \lambda(x)
$$

and just as in the proof of the previous proposition, we get that this is equivalent with $a \lambda(x)=\lambda(a x)$ for all $a$ and for all $x$.

We now want to introduce the notion of an inner action. To motivate the definition (definition 4.13 below) let us again first consider an action $\alpha$ of a group $G$ on an algebra $R$ with identity. Then $\alpha$ is called inner if there is a homomorphism $p \rightarrow u_{p}$ from $G$ into the invertible elements of $R$ so that $\alpha_{p}(x)=u_{p} x u_{p}^{-1}$ for all $p \in G$ and $x \in R$. It is no strong restriction to require $u_{e}=1$ (because then $\alpha_{e}=1$ ). If $R$ has no identity, this notion would be too restrictive and it is much more natural to allow homomorphisms from $G$ to $M(R)$.

In the case of a regular multiplier Hopf algebra, we will have to consider therefore homomorphism $\gamma$ of $A$ into $M(R)$. But also here we have to require them to be unital in the following sense.

4.12 Definition A homomorphism $\gamma: A \rightarrow M(R)$ is called unital if $\gamma(A) R=$ $R \gamma(A)=R$.

In [VD3] and [D-VD], we spoke about non-degenerate homomorphisms. Again, for this, we were inspired by the $\mathrm{C}^{*}$-algebra context. But just as for modules, it now seems to be more natural to call this property unital. Indeed, just as for actions, unital homomorphisms have unique extensions to homomorphisms from $M(A)$ to $M(R)$. This extension is still denoted by $\gamma$ and it can be defined by e.g. $\gamma(m)(\gamma(a) x)=\gamma(m a) x$ and $(x \gamma(a)) \gamma(m)=x \gamma(a m)$ whenever $a \in A, m \in M(A)$ and $x \in R$. For this extension we have $\gamma(1)=1$. That is why, also here, we call $\gamma$ unital.

Now, we are ready to give our definition of an inner action.

4.13 Definition Let $(A, \Delta)$ be a regular multiplier Hopf algebra acting on an algebra $R$. We call the action inner if there is a unital homomorphism $\gamma$ : $A \rightarrow M(R)$ so that

$$
a x=\sum \gamma\left(a_{(1)}\right) x \gamma\left(S\left(a_{(2)}\right)\right)
$$

for all $a$ in $A$ and $x \in R$.

Remark that the expression on the right makes sense because, as $\gamma$ is unital, for all $x \in R$ we have $e \in A$ so that $x=\gamma(e) x$. This element $e$ covers $a_{(1)}$.

It is obvious that the adjoint action, as defined in proposition 4.5 , is actually inner (with $\gamma=\iota$ ). 
A natural extension of innerness is the cocycle equivalence of two actions (sometimes called weak equivalence). This type of equivalence was first considered (and appeared very naturally) in the context of actions of locally compact groups on von Neumann algebras (see e.g. [VD1]). It is important in crossed product theory.

To motivate the definition (definition 4.14 below), let us again first have a look at two actions $\alpha$ and $\beta$ of a group on an algebra $R$ with identity. They are called cocycle (or weak) equivalent if there is a map $p \rightarrow u_{p}$ of $G$ into the invertible elements of $R$ satisfying

i) $u_{p q}=u_{p} \alpha_{p}\left(u_{q}\right)$ for all $p, q \in G$,

ii) $\beta_{p}(x)=u_{p} \alpha_{p}(x) u_{p}^{-1}$ for all $p \in G$ and $x \in R$.

Again, it is natural to require $u_{e}=1$. Remark that condition i) is also very natural when $\alpha$ and $\beta$ are actions satisfying ii). Indeed, for $p, q \in G$ we get

$$
\begin{aligned}
\beta_{p q}(x) & =u_{p q} \alpha_{p q}(x) u_{p q}^{-1} \\
& =u_{p} \alpha_{p}\left(u_{q}\right) \alpha_{p}\left(\alpha_{q}(x)\right) \alpha_{p}\left(u_{q}\right)^{-1} u_{p}^{-1} \\
& =u_{p} \alpha_{p}\left(u_{q} \alpha_{q}(x) u_{q}^{-1}\right) u_{p}^{-1} \\
& =\beta_{p}\left(\beta_{q}(x)\right) .
\end{aligned}
$$

In order to formulate this notion in the context of multiplier Hopf algebras, we will have to replace the map $p \rightarrow u_{p}$ by a linear map from $A$ to $M(R)$. We are able to do this since we have already defined the extension of the action of $A$ to $M(R)$. We only give the definition in the case of a Hopf algebra (for reasons we will explain afterwards).

4.14 Definition Let $(A, \Delta)$ be a Hopf algebra, acting on an algebra $R$ in two ways. Denote these two actions by $a \triangleright_{1} x$ and $a \triangleright_{2} x$. We call these two actions cocycle equivalent if there is a linear map $\gamma: A \rightarrow M(R)$ such that $\gamma(1)=1$ and satisfying

i) $\gamma\left(a a^{\prime}\right)=\sum \gamma\left(a_{(1}\right)\left(a_{(2)} \triangleright_{1} \gamma\left(a^{\prime}\right)\right)$

ii) $\sum\left(a_{(1)} \triangleright_{2} x\right) \gamma\left(a_{(2)}\right)=\sum \gamma\left(a_{(1)}\right)\left(a_{(2)} \triangleright_{1} x\right)$

for all $a, a^{\prime} \in A$ and $x \in R$.

There are some difficulties to extend this notion to the case of regular multiplier Hopf algebras. We will need some kind of non-degeneracy condition on $\gamma$ (to replace the condition $\gamma(1)=1$ ), but it is not so clear how to define this since $\gamma$ is no longer assumed to be a homomorphism. It is however possible to give a meaning to the two other reguirements. The second one is easy as the actions are assumed to be unital. To give a meaning to the first condition, we can rewrite it as

$$
\gamma\left(a a^{\prime}\right) x=\sum \gamma\left(a_{(1}\right) a_{(2)} \triangleright_{1}\left(\left(\gamma\left(a^{\prime}\right)\left(S\left(a_{(3)}\right) \triangleright_{1} x\right)\right)\right)
$$

for all $a, a^{\prime} \in A$ and $x \in R$. 


\section{Smash Products}

Let $(A, \Delta)$ be a regular multiplier Hopf algebra and let it act on an algebra $R$ with a non-degenerate product as in the previous section.

By assumption, $R$ is a left $A$-module and we have

$$
a(x y)=\sum\left(a_{(1)} x\right)\left(a_{(2)} y\right)
$$

for all $x, y \in R$ and $a \in A$. If we also think of $R$ as a left R-module, we are led to the following definition.

5.1 Definition Let $V$ be a vector space which is both a left $A$-module and a left $R$-module. We say that $V$ is a covariant $A$ - $R$-module if

$$
a(x v)=\sum\left(a_{(1)} x\right) a_{(2)} v
$$

for all $a \in A, x \in R$ and $v \in V$.

Remark that this formula makes sense because $a_{(1)}$ is covered by $x$. If $V$ is a unital left $A$-module, we can rewrite this formula as

$$
(a x) v=\sum a_{(1)}\left(x S\left(a_{(2)}\right) v\right)
$$

In this case, $a_{(2)}$ is covered by $v$. This reminds us of the formulas for the adjoint action (see example 4.5 and 4.6). These formulas are indeed very natural. If we consider e.g. $R$ not only as a left $A$-module, but also as a left $R$-module, then $R$ becomes a covariant $A-R$-module and the two formulas above are found in the definition 4.1 and lemma 4.2 .

The name comes from the theory of actions of locally compact groups on operator algebras (see e.g. $[\mathrm{P}]$ ). The following examples refers to this original notion of covariant representation (in the simpler setting of a group action).

5.2 Example Let $G$ be a group and assume that $\alpha$ is an action of $G$ on $R$. Let $V$ be a vector space over $\mathbb{C}$. Suppose that $\pi$ is a representation of $R$ on $V$ and that $p \rightarrow u_{p}$ is a representation of $G$ on $V$ satisfying

$$
\pi\left(\alpha_{p}(x)\right)=u_{p} \pi(x) u_{p}^{-1}
$$

for all $x \in R$ and $p \in G$. Such a pair is called a covariant representation of the covariant system $(R, G, \alpha)$ in $[\mathrm{P}]$. Now let $A$ be the group algebra $\mathbb{C} G$ of $G$. Then, the respresentation of $G$ makes $V$ into a $A$-module. Similarly, the representation of $R$ makes $V$ into a $R$-module. And the relation $\pi\left(\alpha_{p}(x)\right)=$ $u_{p} \pi(x) u_{p}^{-1}$ precisely means that $V$ is a covariant $A$-R-module. 
Now, let $V$ be a covariant $A$ - $R$-module. It follows from the definition that

$$
x a x^{\prime} a^{\prime} v=\sum\left(x\left(a_{(1)} x^{\prime}\right)\right)\left(a_{(2)} a^{\prime} v\right)
$$

when $a, a^{\prime} \in A, x, x^{\prime} \in R$ and $v \in V$. This formula suggests a multiplication on the tensor product $R \otimes A$ of the spaces $R$ and $A$ making $V$ into a left ( $R \otimes A$ )-module. This motivates the introduction of the smash product.

5.3 Definition Define a multiplication on $R \otimes A$ by

$$
(x \otimes a)\left(x^{\prime} \otimes a^{\prime}\right)=\sum x\left(a_{(1)} x^{\prime}\right) \otimes a_{(2)} a^{\prime}
$$

whenever $x, x^{\prime} \in R$ and $a, a^{\prime} \in A$.

There is no problem with this definition: $a_{(1)}$ is covered by $x^{\prime}$ and $a_{(2)}$ is covered by $a^{\prime}$.

The product above is given by the map

$$
(m \otimes m)(\iota \otimes \Gamma \otimes \iota)
$$

where $\Gamma: A \otimes R \rightarrow R \otimes A$ is defined by

$$
\Gamma(a \otimes x)=\sum a_{(1)} x \otimes a_{(2)}
$$

and where $m$ is used to denote both the multiplication in $A$ and in $R$, considered as linear maps from $A \otimes A$ to $A$ and $R \otimes R$ to $R$ respectively. The associativity of the product, as well as other properties, can be obtained from properties of $\Gamma$. This method was followed in $[\mathrm{D}-\mathrm{VD}]$ and before in $[\mathrm{VD}-\mathrm{VK}]$. Here we will follow the more direct approach. The calculations one has to make are esssentially the same in both cases. The direct approach has the advantage of being more self-contained. On the other hand, we will encounter the twist map $\Gamma$ later and so, it is good to have in mind the above formula for the product.

We first have to prove the following.

5.4 Lemma The product defined above is associative.

Proof : Take $x, x^{\prime}, x^{\prime \prime} \in R$ and $a, a^{\prime}, a^{\prime \prime} \in A$. Then

$$
\begin{aligned}
\left((x \otimes a)\left(x^{\prime} \otimes a^{\prime}\right)\right)\left(x^{\prime \prime} \otimes a^{\prime \prime}\right) & =\sum\left(\left(x\left(a_{(1)} x^{\prime}\right)\right) \otimes\left(a_{(2)} a^{\prime}\right)\right)\left(x^{\prime \prime} \otimes a^{\prime \prime}\right) \\
& =\sum x\left(a_{(1)} x^{\prime}\right)\left(a_{(2)} a_{(1)}^{\prime} x^{\prime \prime}\right) \otimes a_{(3)} a_{(2)}^{\prime} a^{\prime \prime} \\
& =\sum x\left(a_{(1)}\left(x^{\prime}\left(a_{(1)}^{\prime} x^{\prime \prime}\right)\right)\right) \otimes a_{(2)} a_{(2)}^{\prime} a^{\prime \prime} \\
& =(x \otimes a) \sum x^{\prime}\left(a_{(1)}^{\prime} x^{\prime \prime}\right) \otimes a_{(2)}^{\prime} a^{\prime \prime} \\
& =(x \otimes a)\left(\left(x^{\prime} \otimes a^{\prime}\right)\left(x^{\prime \prime} \otimes a^{\prime \prime}\right)\right) .
\end{aligned}
$$


So, we have made $R \otimes A$ into an associative algebra. In this context, we will use the following notations.

5.5 Notation When we consider $R \otimes A$ with the above product, we will use the notation $R \# A$ instead and elements $x \otimes a$ will be denoted by $x \# a$.

From the motivation above, we see that any covariant $A$ - $R$-module $V$ gives rise to a $R \# A$-module structure on $V$. We will come back to this in proposition 5.11 . In particular, this is the case for the covariant $A$ - $R$-module $R$. The action of $R \# A$ on $R$ is given by $(x \# a) x^{\prime}=x\left(a x^{\prime}\right)$ whenever $a \in A$ and $x, x^{\prime} \in R$. If this module was faithful, we could have used it to define $R \# A$. Unfortunately, in general, this will not be the case. We will however use this module often and in some special cases, it will be faithful.

It is easy to see that, in the case of the example 5.2 , the algebra $R \# A$ is the algebra of functions from $G$ to $R$ with finite support with the twisted convolution product

$$
(\xi \eta)(p)=\sum_{q} \xi(q) \alpha_{q}\left(\eta\left(q^{-1} p\right)\right)
$$

So, $R \# A$ is the crossed product $R \times{ }_{\alpha} G$ in the sense of $[\mathrm{P}]$.

We will now investigate the algebra $R \# A$ further. The first (important) step is the following.

5.6 Lemma The product in $R \# A$ is non-degenerate.

Proof : Suppose that $\sum x_{i} \# a_{i} \in R \# A$ and that $\left(\sum x_{i} \# a_{i}\right)(x \# a)=0$ for all $x \in R$ and $a \in A$. Then, using the definition of the product in $R \# A$ and the non-degeneracy of the product in $A$, we get

$$
\sum x_{i}\left(a_{i(1)} x\right) \# a_{i(2)}=0
$$

for all $x \in R$. Now, we can proceed as before (see the technique used in the proof of proposition 2.2 or in the proof of proposition 3.4 in [VD6]). Apply $\Delta$ and $S$, multiply with $a, a^{\prime}$ and replace $x$ by $a^{\prime \prime} x$ to obtain

$$
\sum x_{i}\left(a_{i(1)} a^{\prime \prime} x\right) \otimes S\left(a_{i(2)}\right) a \otimes a_{i(3)} a^{\prime}=0
$$

for all $a, a^{\prime}, a^{\prime \prime} \in A$ and $x \in R$. Now, we can replace $a^{\prime \prime}$ by $S\left(a_{i(2)}\right) a$ as these elements are forced (by the use of $a$ and $a^{\prime}$ ) to lie in a finite-dimensional space. This gives

$$
\sum x_{i}\left(a_{i(1)} S\left(a_{i(2)}\right) a x\right) \otimes a_{i(3)} a^{\prime}=0
$$

and hence

$$
\sum x_{i}(a x) \otimes a_{i} a^{\prime}=0
$$


for all $a, a^{\prime} \in A$ and $x \in R$. We can again cancel $a^{\prime}$. And because $R$ is unital so that $A R=R$, we can also cancel $a x$ and we obtain $\sum x_{i} \otimes a_{i}=0$.

Now suppose that $(x \# a)\left(\sum x_{i} \# a_{i}\right)=0$ for all $x \in R$ and $a \in A$. This means that

$$
\sum x\left(a_{(1)} x_{i}\right) \otimes a_{(2)} a_{i}=0
$$

for all $x$ and all $a$. If we multiply the second factor from the left with $a^{\prime}$ and use the fact that $(1 \otimes A) \Delta(A)=A \otimes A$, we get that also

$$
\sum x\left(a x_{i}\right) \otimes a^{\prime} a_{i}=0
$$

for all $x \in R$ and $a, a^{\prime} \in A$. We can cancel $x$, cancel $a^{\prime}$ and use the nondegeneracy of the module $R$ to get again $\sum x_{i} \otimes a_{i}=0$.

If $R$ has an identity, then we get

$$
\begin{aligned}
(1 \# a)\left(1 \# a^{\prime}\right) & =\sum\left(a_{(1)} 1\right) \# a_{(2)} a^{\prime} \\
& =\sum \epsilon\left(a_{(1)}\right) 1 \# a_{(2)} a^{\prime} \\
& =1 \# a a^{\prime}
\end{aligned}
$$

and it follows that $a \rightarrow 1 \# a$ is a homomorphism of $A$ into $R \# A$. Similarly, if $A$ has an identity we get

$$
(x \# 1)\left(x^{\prime} \# 1\right)=x\left(1 x^{\prime}\right) \# 1=x x^{\prime} \# 1
$$

and $x \rightarrow x \# 1$ is a homomorphism of $R$ into $R \# A$. If $R$ and $A$ have identities, we get moreover that

$$
\begin{aligned}
& (x \# 1)(1 \# a)=x \# a \\
& (1 \# a)(x \# 1)=\sum a_{(1)} x \# a_{(2)}
\end{aligned}
$$

for all $a \in A$ and $x \in R$. In this case, $1 \otimes 1$ is an identity in $R \# A$.

In the general case, we have similar results (see e.g. the formulas in proposition 5.9) but we have to consider the multiplier algebra $M(R \# A)$ of $R \# A$. This is possible because we have seen that the product in $R \# A$ is non-degenerate. First, we get the following result.

5.7 Proposition There exists a linear map $\pi: A \rightarrow M(R \# A)$ defined by

$$
\begin{aligned}
\pi(a)\left(x^{\prime} \# a^{\prime}\right) & =\sum a_{(1)} x^{\prime} \# a_{(2)} a^{\prime} \\
\left(x^{\prime} \# a^{\prime}\right) \pi(a) & =x \# a^{\prime} a
\end{aligned}
$$

whenever $a, a^{\prime} \in A$ and $x \in R$. This map is a unital algebra homomorphism. 
Proof : We first check that the above formulas give a well-defined element $\pi(a)$ in $M(R \# A)$. Indeed, for all $a, a^{\prime}, a^{\prime \prime} \in A$ and $x^{\prime}, x^{\prime \prime} \in R$ we get

$$
\begin{aligned}
\left(x^{\prime \prime} \# a^{\prime \prime}\right)\left(\sum a_{(1)} x^{\prime} \# a_{(2)} a^{\prime}\right) & =\sum x^{\prime \prime}\left(a_{(1)}^{\prime \prime} a_{(1)} x^{\prime}\right) \# a_{(2)}^{\prime \prime} a_{(2)} a^{\prime} \\
& =\left(x \# a^{\prime \prime} a\right)\left(x^{\prime} \# a^{\prime}\right)
\end{aligned}
$$

so that indeed we have the formula

$$
\left(x^{\prime \prime} \# a^{\prime \prime}\right)\left(\pi(a)\left(x^{\prime} \# a^{\prime}\right)\right)=\left(\left(x^{\prime \prime} \# a^{\prime \prime}\right) \pi(a)\right)\left(x^{\prime} \# a^{\prime}\right) .
$$

That $\pi$ is a homomorphism follows easily from the formula $\left(x^{\prime} \# a^{\prime}\right) \pi(a)=$ $x^{\prime} \# a^{\prime} a$.

Let us now prove that $\pi$ is unital. We clearly have $(R \# A) \pi(A)=R \# A$ because $A^{2}=A$. On the other hand, because $\Delta(A)(1 \otimes A)=A \otimes A$, we also get $\pi(A)(R \# A)=R \# A$. This completes the proof.

Similarly, we get the following.

5.8 Proposition There exists a linear map $\pi: R \rightarrow M(R \# A)$ given by

$$
\begin{aligned}
& \pi(x)\left(x^{\prime} \# a^{\prime}\right)=x x^{\prime} \# a^{\prime} \\
& \left(x^{\prime} \# a^{\prime}\right) \pi(x)=\sum x^{\prime}\left(a_{(1)} x\right) \# a_{(2)}
\end{aligned}
$$

whenever $a \in A$ and $x, x^{\prime} \in R$. This map is an algebra homomorphism of $R$ into $M(R \# A)$. If $R^{2}=R$, then $\pi$ is unital.

Proof : For all $a^{\prime}, a^{\prime \prime} \in A$ and $x, x^{\prime}, x^{\prime \prime} \in R$ we get

$$
\begin{aligned}
\left(x^{\prime \prime} \# a^{\prime \prime}\right)\left(x x^{\prime} \# a^{\prime}\right) & =\sum x^{\prime \prime}\left(a_{(1)}^{\prime \prime}\left(x x^{\prime}\right)\right) \# a_{(2)} a^{\prime} \\
& =\sum x^{\prime \prime}\left(a_{(1)}^{\prime \prime} x\right)\left(a_{(2)}^{\prime \prime} x^{\prime}\right) \# a_{(3)} a^{\prime} \\
& =\left(\sum x^{\prime \prime}\left(a_{(1)}^{\prime \prime} x\right) \# a_{(2)}^{\prime \prime}\right)\left(x^{\prime} \# a^{\prime}\right) .
\end{aligned}
$$

so that

$$
\left(x^{\prime \prime} \# a^{\prime \prime}\right)\left(\pi(x)\left(x^{\prime} \# a^{\prime}\right)\right)=\left(\left(x^{\prime \prime} \# a^{\prime \prime}\right) \pi(x)\right)\left(x^{\prime} \# a^{\prime}\right)
$$

and $\pi(x)$ is a well-defined element in $M(R \# A)$.

Again from the first formula it follows that $\pi$ is a homomorphism.

When $R^{2}=R$ we get $\pi(R)(R \# A)=R \# A$.

On the other hand, if we replace $x^{\prime}$ by $a^{\prime} x^{\prime}$ and use that $A R=R$ and that $\Delta(A)(A \otimes 1)=A \otimes A$, we get that $(R \# A) \pi(R)$ is also equal to $R^{2} \# A$. So again, when $R^{2}=R$ we get $(R \# A) \pi(R)=R \# A$.

If we don't have $R^{2}=R$, we just get $(R \# A) \pi(R)=\pi(R)(R \# A)=R^{2} \# A$. Now, in general we need unital homomorphisms into the multiplier algebra in order to 
be able to extend them to multiplier algebras. So, we have a slight problem here when $R^{2} \neq R$. We will see after the next result that there is a way around it in this case.

We first prove some formulas that we had earlier in the case of algebras with identity.

5.9 Proposition When $\pi: A \rightarrow M(R \# A)$ and $\pi: R \rightarrow M(R \# A)$ are defined as in the previous propositions, we have

$$
\begin{aligned}
& \pi(x) \pi(a)=x \# a \\
& \pi(a) \pi(x)=\sum a_{(1)} x \# a_{(2)}
\end{aligned}
$$

for all $a \in A$ and $x \in R$.

Proof : Take $a, a^{\prime} \in A$ and $x, x^{\prime} \in R$. Then we get

$$
\begin{aligned}
\pi(x) \pi(a)\left(x^{\prime} \# a^{\prime}\right) & =\pi(x) \sum a_{(1)} x^{\prime} \# a_{(2)} a^{\prime} \\
& =\sum x\left(a_{(1)} x^{\prime}\right) \# a_{(2)} a^{\prime} \\
& =(x \# a)\left(x^{\prime} \# a^{\prime}\right)
\end{aligned}
$$

and

$$
\begin{aligned}
\pi(a) \pi(x)\left(x^{\prime} \# a^{\prime}\right) & =\pi(a)\left(x x^{\prime} \# a^{\prime}\right) \\
& =\sum a_{(1)}\left(x x^{\prime}\right) \# a_{(2)} a^{\prime} \\
& =\sum\left(a_{(1)} x\right)\left(a_{(2)} x^{\prime}\right) \# a_{(3)} a^{\prime} \\
& =\left(\sum a_{(1)} x \# a_{(2)}\right)\left(x^{\prime} \# a^{\prime}\right) .
\end{aligned}
$$

It follows that $R \# A=\pi(R) \pi(A)=\pi(A) \pi(R)$. This implies e.g. that we can extend $\pi$ from $R$ to $M(R)$ (also when $R^{2} \neq R$ ). Indeed, given $m \in M(R)$ we define $\pi(m)$ in $M(R \# A)$ by $\pi(m)(\pi(x) \pi(a))=\pi(m x) \pi(a)$ and $(\pi(a) \pi(x)) \pi(m)=\pi(a) \pi(x m)$. We will need this in section 7 . The map

$$
x \otimes a \rightarrow \sum a_{(1)} x \otimes a_{(2)}
$$

from $R \otimes A$ to itself will play a fundamental rôle further. We see here that it determines the commutation rules between elements of $\pi(A)$ and $\pi(R)$.

We now are ready to prove a universal property (related with these commutation rules).

5.10 Proposition Let $(A, \Delta)$ be a regular multiplier Hopf algebra, acting on an algebra $R$. Suppose that $C$ is an algebra over $\mathbb{C}$ and that there exists homomorphisms $\pi: A \rightarrow M(C)$ and $\pi: R \rightarrow M(C)$ such that

$$
\pi(a) \pi(x)=\sum \pi\left(a_{(1)} x\right) \pi\left(a_{(2)}\right)
$$


for all $a \in A$ and $x \in R$. Then there is a homomorphism $\pi: R \# A \rightarrow M(C)$ such that

$$
\pi(x \# a)=\pi(x) \pi(a) .
$$

Proof : Define $\pi: R \# A \rightarrow M(C)$ by $\pi(x \# a)=\pi(x) \pi(a)$. To verify that $\pi$ is a homomorphism, take $a, a^{\prime} \in A$ and $x, x^{\prime} \in R$. We have

$$
\begin{aligned}
\pi\left((x \# a)\left(x^{\prime} \# a^{\prime}\right)\right) & =\pi\left(\sum\left(x\left(a_{(1)} x^{\prime}\right) \# a_{(2)} a^{\prime}\right)\right) \\
& =\sum \pi\left(x\left(a_{(1)} x^{\prime}\right)\right) \pi\left(a_{(2)} a^{\prime}\right) \\
& =\sum \pi(x) \pi\left(a_{(1)} x^{\prime}\right) \pi\left(a_{(2)}\right) \pi\left(a^{\prime}\right) \\
& =\pi(x) \pi(a) \pi\left(x^{\prime}\right) \pi\left(a^{\prime}\right) \\
& =\pi(x \# a) \pi\left(x^{\prime} \# a^{\prime}\right) .
\end{aligned}
$$

If we assume that $\pi$ is unital on $A$, i.e. $\pi(A) C=C$ and $C \pi(A)=C$, then we can rewrite the condition. Indeed, for all $a, a^{\prime} \in A$ and $x \in R$ and $b \in C$ we have

$$
\begin{aligned}
\sum \pi\left(a_{(1)}\right) \pi(x) \pi\left(S\left(a_{(2)}\right) a^{\prime}\right) b & =\sum \pi\left(a_{(1)} x\right) \pi\left(a_{(2)} S\left(a_{(3)}\right) a^{\prime}\right) b \\
& =\pi(a x) \pi\left(a^{\prime}\right) b
\end{aligned}
$$

and since $\pi(A) C=C$ we can interprete this formula as

$$
\pi(a x)=\sum \pi\left(a_{(1)}\right) \pi(x) \pi\left(S\left(a_{(2)}\right)\right)
$$

The difficulty with the last expression is that non of the $a_{(k)}$ seem to be covered. This sum must be considered in the strict topology on $M(C)$. Indeed, if we multiply to the left or to the right with an element in $B$, we obtain a covering for $a_{(1)}$ or for $a_{(2)}$ and we end up with finite sums.

Now we look at a similar result for covariant modules. Suppose we have a left $R \# A$-module $V$ which is unital. Then, we know that we can extend $V$ to a left $M(R \# A)$-module. Because we have our homomorphisms $\pi: A \rightarrow M(R \# A)$ and $\pi: R \rightarrow M(R \# A)$, we have actions of $A$ and $R$ on $V$. From the formula

$$
\pi(a) \pi(x)=\sum \pi\left(a_{(1)} x\right) \pi\left(a_{(2)}\right)
$$

we get, in module formulation

$$
a(x v)=\sum\left(a_{(1)} x\right) a_{(2)} v .
$$

This precisely means that $V$ is a covariant $A$-R-module. Remark that $V$ is a unital $A$-module as $A V=\pi(A)(R \# A) V=(R \# A) V=V$. 
We now prove the converse (which we announced already in the beginning of this section).

5.11 Proposition Suppose that $V$ is a covariant $A$ - $R$-module. Then $V$ is also a $R \# A$-module and the action of $R \# A$ is given by $(x \# a) v=x(a v)$ for all $x \in R, a \in A$ and $v \in V$.

Proof : Take $a, a^{\prime} \in A, x, x^{\prime} \in R$ and $v \in V$. Then we get

$$
\begin{aligned}
\left((x \# a)\left(x^{\prime} \# a^{\prime}\right)\right) v & =\left(\sum x\left(a_{(1)} x^{\prime}\right) \# a_{(2)} a^{\prime}\right) v \\
& =\sum\left(x\left(a_{(1)} x^{\prime}\right)\right)\left(\left(a_{(2)} a^{\prime}\right) v\right) \\
& =\sum x\left(a_{(1)} x^{\prime}\right) a_{(2)} a^{\prime} v \\
& =x\left(a\left(x^{\prime} a^{\prime} v\right)\right) \\
& =(x \# a)\left(\left(x^{\prime} \# a^{\prime}\right) v\right)
\end{aligned}
$$

and we get an action of $R \# A$ on $V$.

When is the action unital ? If we assume that $V$ is unital, both for $A$ and for $R$, then we get

$$
(R \# A) V=R(A V)=R V=V .
$$

Conversely, if $(R \# A) V=V$, then $R A V=V$ and $A R V=V$ and these imply that $R V=V$ and $A V=V$.

Because $R$ is not assumed to have local units (in the sense of proposition 2.2), we also want to look at the non-degeneracy of the module actions (in the sense of proposition 3.2). Suppose first that $V$ is a non-degenerate $R$-module. Let $v \in V$ be given and assume that $(x \# a) v=0$ for all $a \in A$ and $x \in R$. This means that $x a v=0$ for all $x \in R$ and $a \in A$. Because $V$ is assumed to be a non-degenerate $R$-module, it follows that $a v=0$ for all $a \in A$. Because $V$ is automatically a nondegenerate $A$-module, we get $v=0$. Hence, $V$ is also a non-degenerate $(R \# A)$ module. Conversely, assume that $V$ is a non-degenerate $(R \# A)$-module. Again take any $v \in V$ and assume that $x v=0$ for all $x \in R$. Then $a x v=0$ for all $x \in R$ and $a \in A$. Using the twist map, we get also that $x a v=0$ for all $x \in R$ and $a \in A$. Hence, we must have $v=0$.

So, we get a one-to-one correspondence between unital left $R \# A$-modules and covariant $R$ - $A$-modules with unital actions. And if one is non-degenerate, so is the other. This result is similar to a well-known result for actions of locally compact groups on $\mathrm{C}^{*}$-algebras (see section 7.6 in $[\mathrm{P}]$ ).

\section{INNER AND EQUIVALENT ACTIONS}


We will first show that the smash product is trivial when the action is inner. Recall that the action of $A$ on $R$ is called inner if there is a unital homomorphism $\gamma: A \rightarrow$ $M(R)$ such that

$$
a x=\sum \gamma\left(a_{(1)}\right) x \gamma\left(S\left(a_{(2)}\right)\right)
$$

for all $a \in A$ and $x \in R$ (cf. definition 4.13).

5.12 Proposition If the action of $A$ on $R$ is inner, then $R \# A$ is isomorphic with $R \otimes A$, now considered with the usual tensor product algebra structure.

Proof : Define $\varphi: R \# A \rightarrow R \otimes A$ by

$$
\varphi(x \# a)=\sum x \gamma\left(a_{(1)}\right) \otimes a_{(2)} .
$$

We first show that $\varphi$ is well-defined. Because we assume $\gamma$ to be unital, we can write $x$ as $\sum x_{i} \gamma\left(a_{i}\right)$. Therefore, we see that $a_{(1)}$ will be covered in the above definition. Moreover, if $\sum x_{i} \gamma\left(a_{i}\right)=0$ then

$$
\sum x_{i} \gamma\left(a_{i} a_{(1)}\right) \otimes a_{(2)} a^{\prime}=\sum x_{i} \gamma\left(a_{i}\right) \gamma\left(a_{(1)}\right) \otimes a_{(2)} a^{\prime}=0
$$

for all $a^{\prime}$ so that also

$$
\sum x_{i} \gamma\left(a_{i} a_{(1)}\right) \otimes a_{(2)}=0
$$

This means that $\varphi$ is well-defined.

Similarly, we can define $\psi: R \otimes A \rightarrow R \# A$ by

$$
\psi(x \otimes a)=\sum x \gamma\left(S\left(a_{(1)}\right)\right) \otimes a_{(2)} .
$$

An easy calculation will show that $\varphi \psi=\psi \varphi=\iota$.

Also, for all $a, a^{\prime} \in A$ and $x, x^{\prime} \in R$ we have

$$
\begin{aligned}
\varphi\left((x \# a)\left(x^{\prime} \# a^{\prime}\right)\right) & =\varphi\left(\sum x\left(a_{(1)} x^{\prime}\right) \# a_{(2)} a^{\prime}\right) \\
& =\sum x\left(a_{(1)} x^{\prime}\right) \gamma\left(a_{(2)} a_{(1)}^{\prime}\right) \otimes a_{(3)} a_{(2)}^{\prime} \\
& =\sum x \gamma\left(a_{(1)}\right) x^{\prime} \gamma\left(a_{(1)}^{\prime}\right) \otimes a_{(2)} a_{(2)}^{\prime} \\
& =\varphi(x \# a) \varphi\left(x^{\prime} \# a^{\prime}\right) .
\end{aligned}
$$

This proves the result.

Next, we will extend the above result to the case of equivalent actions. We can only do this in the case of an action of a Hopf algebra because we only have defined cocycle equivalent actions properly in that case. Recall that two actions $\triangleright_{1}$ and $\triangleright_{2}$ are called (cocycle) equivalent (see definition 4.14) if there is a linear map $\gamma: A \rightarrow M(R)$ satisfying $\gamma(1)=1$ and 
i) $\gamma\left(a a^{\prime}\right)=\sum \gamma\left(a_{(1}\right)\left(a_{(2)} \triangleright_{1} \gamma\left(a^{\prime}\right)\right)$

ii) $\sum\left(a_{(1)} \triangleright_{2} x\right) \gamma\left(a_{(2)}\right)=\sum \gamma\left(a_{(1)}\right)\left(a_{(2)} \triangleright_{1} x\right)$

for all $a, a^{\prime} \in A$ and $x \in R$. We get the following result.

5.13 Proposition If two actions $\triangleright_{1}$ and $\triangleright_{2}$ of a Hopf algebra $A$ on $R$ are cocycle equivalent, then the corresponding smash products $R \#_{1} A$ and $R \#_{2} A$ are isomorphic.

Proof : Define $\varphi: R \#_{2} A \rightarrow R \#_{1} A$ by

$$
\varphi\left(x \#_{2} a\right)=\sum x \gamma\left(a_{(1)}\right) \#_{1} a_{(2)}
$$

whenever $x \in R$ and $a \in A$. Here, this map will be well-defined since we are working with a Hopf algebra. We claim that $\varphi$ has an inverse $\psi$ given by

$$
\psi\left(x \#_{1} a\right)=\sum x\left(a_{(1)} \triangleright_{1} \gamma\left(S\left(a_{(2)}\right)\right)\right) \#_{2} a_{(3)} .
$$

We get

$$
\begin{aligned}
\psi \varphi\left(x \#_{2} a\right) & =\psi\left(\sum x \gamma\left(a_{(1)}\right) \#_{1} a_{(2)}\right) \\
& =\sum x \gamma\left(a_{(1)}\right)\left(a_{(2)} \triangleright_{1} \gamma\left(S\left(a_{(3)}\right)\right)\right) \#_{2} a_{(4)} \\
& =\sum x \gamma\left(a_{(1)} S a_{(2)}\right) \#_{2} a_{(3)} \\
& =x \#_{2} a
\end{aligned}
$$

because $\gamma(1)=1$. Similarly we obtain that

$$
\begin{aligned}
\varphi \psi\left(x \#_{1} a\right) & =\varphi\left(\sum x\left(a_{(1)} \triangleright_{1} \gamma\left(S\left(a_{(2)}\right)\right)\right) \#_{2} a_{(3)}\right) \\
& =\sum x\left(a_{(1)} \triangleright_{1} \gamma\left(S\left(a_{(2)}\right)\right) \gamma\left(a_{(3)}\right)\right) \#_{1} a_{(4)} \\
& =\sum x\left(a_{(1)} \triangleright_{1}\left(\gamma\left(S\left(a_{(3)}\right)\right)\left(S\left(a_{(2)}\right)\right) \triangleright_{1} \gamma\left(a_{(4)}\right)\right)\right) \#_{1} a_{(5)} \\
& =\sum x\left(a_{(1)} \triangleright_{1} \gamma\left(S\left(a_{(2)} a_{(3)}\right)\right)\right) \#_{1} a_{(4)} \\
& =x \#_{1} a .
\end{aligned}
$$

Now, we verify that we have a homomorphism of algebras. Take $x, x^{\prime} \in R$ and $a, a^{\prime} \in A$. Then

$$
\begin{aligned}
\varphi\left(\left(x \#_{2} a\right)\left(x^{\prime} \#_{2} a^{\prime}\right)\right) & =\varphi\left(\sum x\left(a_{(1)} \triangleright_{2} x^{\prime}\right) \#_{2} a_{(2)} a^{\prime}\right) \\
& =\sum x\left(a_{(1)} \triangleright_{2} x^{\prime}\right) \gamma\left(a_{(2)} a_{(1)}^{\prime}\right) \#_{1} a_{(3)} a_{(2)}^{\prime} \\
& =\sum x\left(a_{(1) \triangleright_{2}} x^{\prime}\right) \gamma\left(a_{(2)}\right)\left(a_{(3)} \triangleright_{1} \gamma\left(a_{(1)}^{\prime}\right)\right) \#_{1} a_{(4)} a_{(2)}^{\prime} \\
& =\sum x \gamma\left(a_{(1)}\left(a_{(2)} \triangleright_{1} x^{\prime}\right)\left(a_{(3)} \triangleright_{1} \gamma\left(a^{\prime}\right)\right) \#_{1} a_{(4)} a_{(2)}^{\prime} .\right.
\end{aligned}
$$


On the other hand

$$
\begin{aligned}
\varphi\left(x \#_{2} a\right) \varphi\left(x^{\prime} \#_{2} a^{\prime}\right) & =\sum\left(x \gamma\left(a_{(1)}\right) \#_{1} a_{(2)}\right)\left(x^{\prime} \gamma\left(a_{(1)}^{\prime}\right) \#_{1} a_{(2)}^{\prime}\right) \\
& =\sum x \gamma\left(a_{(1)}\right)\left(a_{(2)} \triangleright_{1}\left(x^{\prime} \gamma\left(a_{(1)}^{\prime}\right)\right)\right) \#_{1} a_{(3)} a_{(2)}^{\prime} \\
& =\sum x \gamma\left(a_{(1)}\left(a_{(2)} \triangleright_{1} x^{\prime}\right)\left(a_{(3)} \triangleright_{1} \gamma\left(a^{\prime}\right)\right) \#_{1} a_{(4)} a_{(2)}^{\prime} .\right.
\end{aligned}
$$

and this is precisely $\varphi\left(\left(x \#_{2} a\right)\left(x^{\prime} \#_{2} a^{\prime}\right)\right)$.

As we have mentioned already in the previous section, where we introduced this notion of equivalence, it will probably be not so easy to extend this to the multiplier Hopf algebra case but it should be possible, provided the right non-degeneracy condition on $\gamma$ is assumed. In any case, the proof might become quite involved.

\section{Special Cases arising from Dual Pairs}

In this section, we will consider some special cases, coming from a dual pair of regular multiplier Hopf algebras (in the sense of [D-VD]). In the case of the pairing between an algebraic quantum group $A$ and its dual $\hat{A}$, we will be able to give an explicit (and simple) form of the smash product. This case is close to the finitedimensional case.

The results that we obtain here will not only serve as an illustration of the results of the previous sections, but will also be basic for the duality, proved and discussed in the next section.

Let us first recall the notion of a pairing between two regular multiplier Hopf algebas (as introduced in [D-VD]).

Start with two regular multiplier Hopf algebras $A$ and $B$ and a non-degenerate bilinear from $\langle$,$\rangle from A \times B$ to $\mathbb{C}$. For all $a \in A$ and $b \in B$ we can define an element $a \triangleright b$ in $M(B)$ by

$$
\begin{aligned}
& (a \triangleright b) b^{\prime}=\sum\left\langle a, b_{(2)}\right\rangle b_{(1)} b^{\prime} \\
& b^{\prime}(a \triangleright b)=\sum\left\langle a, b_{(2)}\right\rangle b^{\prime} b_{(1)}
\end{aligned}
$$

whenever $b^{\prime} \in B$. Let us now assume that the pairing is such that $a \triangleright b \in B$ for all $a \in A$ and $b \in B$. Then, it makes sense to require that

$$
\left\langle a^{\prime}, a \triangleright b\right\rangle=\left\langle a^{\prime} a, b\right\rangle
$$

for all $a, a^{\prime} \in A$ and $b \in B$. This essentially means that the product in $A$ is dual to the coproduct in $B$. Then, for all $a, a^{\prime}, a^{\prime \prime} \in A$ and $b \in B$ we will have

$$
\begin{aligned}
\left\langle a,\left(a^{\prime} a^{\prime \prime}\right) \triangleright b\right\rangle & =\left\langle a\left(a^{\prime} a^{\prime \prime}\right), b\right\rangle \\
& =\left\langle a a^{\prime}, a^{\prime \prime} \triangleright b\right\rangle \\
& =\left\langle a, a^{\prime} \triangleright(a \triangleright b)\right\rangle
\end{aligned}
$$


and because we have taken our pairing to be non-degenerate, we get

$$
\left(a^{\prime} a^{\prime \prime}\right) \triangleright b=a^{\prime} \triangleright(a \triangleright b)
$$

Therefore, $B$ is a left $A$-module.

This observation was the main motivation for the introduction of the notion of a prepairing in $[\mathrm{D}-\mathrm{VD}]$. In fact, we impose four conditions like the ones above. We require, for all $a \in A$ and $b \in B$,

$$
\begin{aligned}
& \sum\left\langle a_{(1)}, b\right\rangle a_{(2)} \in A \\
& \sum\left\langle a_{(2)}, b\right\rangle a_{(1)} \in A \\
& \sum\left\langle a, b_{(1)}\right\rangle b_{(2)} \in B \\
& \sum\left\langle a, b_{(2)}\right\rangle b_{(1)} \in B
\end{aligned}
$$

and for all $a, a^{\prime} \in A$ and $b, b^{\prime} \in B$,

$$
\begin{aligned}
& \left\langle\sum\left\langle a_{(1)}, b\right\rangle a_{(2)}, b^{\prime}\right\rangle=\left\langle a, b b^{\prime}\right\rangle \\
& \left\langle\sum\left\langle a_{(2)}, b\right\rangle a_{(1)}, b^{\prime}\right\rangle=\left\langle a, b^{\prime} b\right\rangle \\
& \left\langle a^{\prime}, \sum\left\langle a, b_{(1)}\right\rangle b_{(2)}\right\rangle=\left\langle a a^{\prime}, b\right\rangle \\
& \left\langle a^{\prime}, \sum\left\langle a, b_{(2)}\right\rangle b_{(1)}\right\rangle=\left\langle a^{\prime} a, b\right\rangle .
\end{aligned}
$$

It follows that four modules are involved. We have that $B$ is a left and a right $A$-module (in fact, an $A$-bimodule) and that $A$ is a left and a right $B$-module (also a $B$-bimodule). We will denote the left actions by $\triangleright$ and the right actions by $\triangleleft$ (as in $[\mathrm{D}-\mathrm{VD}])$.

In proposition 2.8 of [D-VD], we have shown that all of these modules are unital (in the sense of definition 3.1 of this paper) if only one of them is unital. This is the case when the prepairing is a pairing (definition 2.9 of [D-VD]).

Remark that the fact that these actions are unital also implies that e.g. in the expression

$$
\sum\left\langle a_{(1)}, b\right\rangle a_{(2)}
$$

the element $a_{(1)}$ is also covered by $b$ in the following sense. Take an element $e \in A$ such that $e \triangleright b=b$ (cf. the remark before proposition 3.3). Then we will have

$$
\sum\left\langle a_{(1)}, b\right\rangle a_{(2)}=\sum\left\langle a_{(1)} e, b\right\rangle a_{(2)}
$$

so that $a_{(1)}$ is covered in the usual sense. This covering of $a_{(1)}$ by $b$ through the pairing in turn implies that

$$
\sum\left\langle a_{(1)}, b\right\rangle a_{(2)} \in A
$$


for all $a \in A$ and $b \in B$. This shows that the axioms for a pairing are not independent from each other. These observations are important for the formulas and calculations further in this paper.

In what follows, we consider a dual pair $(A, B)$ of regular multiplier Hopf algebras in the sense of definition 2.9 of [D-VD] (as discussed before).

In the first proposition of this section, we show that $A$ is a $B$-module algebra and that $B$ is a $A$-module algebra. The result indicates once more that the notion of a pairing, as introduced in [D-VD] is indeed a natural one (and not only for the treatment of the quantum double).

6.1 Proposition The left action of $A$ on $B$ defined by

$$
a \triangleright b=\sum\left\langle a, b_{(2)}\right\rangle b_{(1)}
$$

makes $B$ into a left $A$-module algebra (in the sense of definition 4.1).

Proof : We just have to show that

$$
a \triangleright\left(b b^{\prime}\right)=\sum\left(a_{(1)} \triangleright b\right)\left(a_{(2)} \triangleright b^{\prime}\right)
$$

for all $a \in A$ and $b, b^{\prime} \in B$. Now, choose $e \in A$ so that $b=e \triangleright b$. Take also $a^{\prime} \in A$. Then

$$
\begin{aligned}
\left\langle a^{\prime}, \sum\left(a_{(1)} \triangleright b\right)\left(a_{(2)} \triangleright b^{\prime}\right)\right\rangle & =\sum\left\langle a^{\prime},\left(\left(a_{(1)} e\right) \triangleright b\right)\left(a_{(2)} \triangleright b^{\prime}\right)\right\rangle \\
& =\sum_{(a)}\left\langle\sum_{\left(a^{\prime}\right)}\left\langle a_{(1)}^{\prime},\left(a_{(1)} e\right) \triangleright b\right\rangle a_{(2)}^{\prime}, a_{(2)} \triangleright b^{\prime}\right\rangle \\
& =\sum\left\langle a_{(1)}^{\prime} a_{(1)} e, b\right\rangle\left\langle a_{(2)}^{\prime} a_{(2)}, b^{\prime}\right\rangle \\
& =\left\langle\sum\left\langle\left(a a^{\prime}\right)_{(1)}, b\right\rangle\left(a a^{\prime}\right)_{(2)}, b^{\prime}\right\rangle \\
& =\left\langle a^{\prime} a, b b^{\prime}\right\rangle=\left\langle a^{\prime}, a \triangleright\left(b b^{\prime}\right)\right\rangle .
\end{aligned}
$$

So, loosely speaking, $B$ is a left $A$-module because the product in $A$ is dual to the coproduct on $B$ and $B$ is a left $A$-module algebra because the product in $B$ is dual to the coproduct on $A$.

Because we have an action of $A$ on $B$, we can define the smash product $B \# A$. Similarly, we have an action of $B$ on $A$ given by

$$
b \triangleright a=\sum\left\langle a_{(2)}, b\right\rangle a_{(1)}
$$

whenever $a \in A$ and $b \in B$. This will yield another smash product $A \# B$. We will now study these algebras and the relation between the two. 
First, observe that it is an immediate consequence of the definitions that the product in $B \# A$ is given by the formula

$$
(b \# a)\left(b^{\prime} \# a^{\prime}\right)=\sum\left\langle a_{(1)}, b_{(2)}^{\prime}\right\rangle b b_{(1)}^{\prime} \# a_{(2)} a^{\prime}
$$

Remark that there is no problem with this formula as $b_{(1)}^{\prime}$ and $a_{(2)}$ are both covered. In the beginning of section 5, we have seen that, in general, there is a natural action of $R \# A$ on $R$ when $R$ is a left $A$-module algebra. The action is given by $(x \# a) x^{\prime}=x\left(a x^{\prime}\right)$ whenever $a \in A$ and $x, x^{\prime} \in R$. This action need not be faithful. In this special case however, we do have a faithful action:

6.2 Proposition The space $B$ is made into a left $(B \# A)$-module by

$$
(b \# a) b^{\prime}=b\left(a \triangleright b^{\prime}\right)
$$

whenever $a \in A$ and $b, b^{\prime} \in B$. This action of $B \# A$ is faithful.

Proof : It is straightforward to check that $B$ is a left $(B \# A)$-module for the action defined above. This was shown already in section 5 . To show that the module is faithful, let $\sum b_{i} \# a_{i} \in B \# A$ and assume that

$$
\sum\left(b_{i} \# a_{i}\right) b^{\prime}=\sum\left\langle a_{i}, b_{(2)}^{\prime}\right\rangle b_{i} b_{(1)}^{\prime}=0
$$

for all $b^{\prime} \in B$. If we multiply with $b^{\prime \prime}$ on the right and if we use that $\Delta(B)(B \otimes$ 1) $=B \otimes B$, we get that

$$
\sum\left\langle a_{i}, b^{\prime}\right\rangle b_{i} b^{\prime \prime}=0
$$

for all $b^{\prime}, b^{\prime \prime} \in B$. We can cancel $b^{\prime \prime}$ because the product in $B$ is non-degenerate and using the non-degeneracy of the pairing, we can conclude that $\sum b_{i} \otimes a_{i}=0$ in $B \otimes A$. This completes the proof.

Compare this result with lemma 9.4.2 of [M2] where the faithfulness of the map $\lambda$ follows from the bijectivity of the antipode.

Related with this proposition is the following characterization of the algebra $B \# A$.

6.3 Proposition Consider the algebra $C$ generated by the algebras $A$ and $B$ subject to the following commutation relations

$$
a b=\sum\left\langle a_{(1)}, b_{(2)}\right\rangle b_{(1)} a_{(2)}
$$

whenever $a \in A$ and $b \in B$. Then $b \# a \rightarrow b a$ gives an injective homomorphism of $B \# A$ into $C$.

Proof : First remark that

$$
\sum\left\langle a_{(1)}, b_{(2)}\right\rangle a_{(2)} \otimes b_{(1)}
$$


is defined within $A \otimes B$ (see proposition 2.8 of [D-VD]). It follows that the algebra $C$ is well-defined. We have an action of $A$ and an action of $B$ on $B$. Moreover, we have

$$
\begin{aligned}
a \triangleright\left(b b^{\prime}\right) & =\sum\left(a_{(1)} \triangleright b\right)\left(a_{(2)} \triangleright b^{\prime}\right) \\
& =\sum\left\langle a_{(1)}, b_{(2)}\right\rangle b_{(1)}\left(a_{(2)} \triangleright b^{\prime}\right)
\end{aligned}
$$

for all $a \in A$ and $b, b^{\prime} \in B$. But this precisely means that these two actions of $A$ and $B$ satisfy the defining commutation rules for $C$ and therefore, these two actions yield an action of $C$ on $B$. Now, since $B$ is a faithfull $(B \# A)$-module for the action given by $(b \# a) b^{\prime}=b\left(a \triangleright b^{\prime}\right)$, we see that the map $b \# a \rightarrow b a$ gives indeed an injective homomorphism of $B \# A$ into $C$.

Remark that the result above is naturally related with the universal property of $R \# A$ as proved in 5.10 .

The commutation rules in 6.3 can also be expressed as

$$
b a=\sum\left\langle S^{-1} a_{(1)}, b_{(2)}\right\rangle a_{(2)} b_{(1)} .
$$

This can easily be verified and in fact, it follows from the fact that the maps

$$
\begin{aligned}
& a \otimes b \rightarrow \sum\left\langle a_{(1)}, b_{(2)}\right\rangle a_{(2)} \otimes b_{(1)} \\
& a \otimes b \rightarrow \sum\left\langle S^{-1} a_{(1)}, b_{(2)}\right\rangle a_{(2)} \otimes b_{(1)}
\end{aligned}
$$

are each others inverses (see also proposition 2.8 in [D-VD]).

So, $B \# A$ can be considered as the span of the elements $a b$ in the algebra generated by $A$ and $B$ subject to the commutation rules above. Similarly, the algebra $A \# B$ can be characterized as the span of the elemens $a b$ in the algebra generated by $A$ and $B$ subject to the commutation relations

$$
b a=\sum\left\langle a_{(2)}, b_{(1)}\right\rangle a_{(1)} b_{(2)}
$$

whenever $a \in A$ and $b \in B$.

The commutation rules above are of the same nature as the Heisenberg commutation rules (e.g. in the case of the pairing between an abelian group $G$ and its dual group $\hat{G}$ ). Therefore, the algebra $C$ in this proposition is sometimes called the Heisenberg double (cf. [M2]). The quantum double (of Drinfel'd) on the other hand can be characterized in a similar way, but by using different commutation rules (see $[\mathrm{D}-\mathrm{VD}])$. In the first case, the commutation rules are related with the Pentagon equation while in the second case, they are related with the Yang-Baxter equation (see e.g. [VD2]).

Using proposition 6.3 , it becomes very easy to get the following relation between $B \# A$ and $A \# B$. 
6.4 Proposition The map $b \# a \rightarrow S^{-1} a \# S b$ defines a anti-isomorphism of $B \# A$ with $A \# B$.

Proof : It is straighforward to check this result directly. On the other hand, one can also easily verify that this map will convert the commutation rules defining $B \# A$ into those defining $A \# B$. Indeed, for all $a \in A$ and $b \in B$ we get

$$
\sum\left\langle\left(S^{-1} a\right)_{(2)},(S b)_{(1)}\right\rangle\left(S^{-1} a\right)_{(1)} \otimes(S b)_{(2)}=\sum\left\langle a_{(1)}, b_{(2)}\right\rangle S^{-1} a_{(2)} \otimes S b_{(1)} .
$$

Compare with proposition 2.14 of [D-VD].

We can use this anti-isomorphism of $B \# A$ with $A \# B$ to obtain a standard right action of $B \# A$ on $A$. Indeed, we can make $A$ into a right $(B \# A)$-module by letting

$$
\begin{aligned}
a^{\prime}(b \# a) & =\left(S^{-1} a \# S b\right) a^{\prime} \\
& =\left(S^{-1} a\right)\left((S b) \triangleright a^{\prime}\right) \\
& =\sum\left\langle a_{(2)}^{\prime}, S b\right\rangle\left(S^{-1} a\right) a_{(1)}^{\prime} .
\end{aligned}
$$

If we replace $a^{\prime}$ by $S^{-1} a^{\prime}$, we find

$$
\left(S^{-1} a^{\prime}\right)(b \# a)=\sum\left\langle a_{(1)}^{\prime}, b\right\rangle S^{-1}\left(a_{(2)}^{\prime} a\right)
$$

and so we get a modified $(B \# A)$-module structure on $A$ given by

$$
a^{\prime}(b \# a)=\left(a^{\prime} \triangleleft b\right) a
$$

whenever $a, a^{\prime} \in A$ and $b \in B$. Of course also this action is faithful. This corresponds with the faithfulness of $\rho$ in lemma 9.4.2 of [M2].

In the next section, where we discuss the duality for actions, we will come back to these two $B \# A$ module structures.

We will specialize to the pairing of an algebraic quantum group $A$ with its dual $\hat{A}$. But before we do this, we will prove a result about fixed points that will be used in the next section.

6.5 Proposition The only fixed points in $M(B)$ for the action of $A$ are multiples of the identity.

Proof : Let $m$ be in $M(B)$ and a fixed point for the action of $A$. We have seen in proposition 4.11 that then

$$
a \triangleright(m b)=m(a \triangleright b)
$$


for all $a \in A$ and $b \in B$. If we apply the counit to this equation, we get

$$
\langle a, m b\rangle=\sum \epsilon\left(m b_{(1)}\right)\left\langle a, b_{(2)}\right\rangle
$$

for all $a$ and $b$. Then we get

$$
m b=\sum \epsilon\left(m b_{(1)}\right) b_{(2)}
$$

for all $b$. Therefore we have for all $b, b^{\prime} \in B$ that

$$
\begin{aligned}
\epsilon(m b) b^{\prime} & =\sum \epsilon\left(m b_{(1)}\right) b_{(2)} S\left(b_{(3)}\right) b^{\prime} \\
& =\sum\left(m b_{(1)}\right)\left(S\left(b_{(2)}\right) b^{\prime}\right. \\
& =\sum m b_{(1)} S\left(b_{(2)}\right) b^{\prime} \\
& =\epsilon(b) m b^{\prime} .
\end{aligned}
$$

So, we get $m=\epsilon(m b) 1$ when $b$ is chosen so that $\epsilon(b)=1$.

Remark that in the proof above, we only have used that $m$ is a left multiplier. In fact, it is for such multipliers that we will need this result in the next section.

Now, in what follows, $A$ is an algebraic quantum group (cf. definition 2.4) and $\hat{A}$ denotes the dual of $A$ (cf. theorem 2.5). The duality between $A$ and $\hat{A}$ makes $(A, \hat{A})$ into a dual pair satisfying the axioms mentioned in the beginning of this section (see [D-VD]). So, we can apply the previous results with $B$ replaced by $\hat{A}$. In particular we have the left action of $\hat{A}$ on $A$ and the corresponding smash product $A \# \hat{A}$. We also have the action of $A \# \hat{A}$ on $A$ given by

$$
(a \# b) a^{\prime}=\sum\left\langle a_{(2)}^{\prime}, b\right\rangle a a_{(1)}^{\prime}
$$

where now $a, a^{\prime} \in A$ and $b \in \hat{A}$.

We get the following result.

6.6 Proposition The algebra $A \# \hat{A}$, as acting on $A$, is the algebra of linear combinations of the (rank one) maps from $A$ to $A$ of the form $a^{\prime} \rightarrow\left\langle a^{\prime}, b\right\rangle a$ where $a \in A$ and $b \in \hat{A}$.

Proof : Let $c \in A$ and consider $b=\varphi(c \cdot)$ in $\hat{A}$ where $\varphi$ is a given left integral on $A$. Then

$$
\begin{aligned}
\sum \varphi\left(c a_{(2)}^{\prime}\right) a a_{(1)}^{\prime} & =\sum \varphi\left(c_{(3)} a_{(2)}^{\prime}\right) a S\left(c_{(1)}\right) c_{(2)} a_{(1)}^{\prime} \\
& =\sum \varphi\left(c_{(2)} a^{\prime}\right) a S\left(c_{(1)}\right) .
\end{aligned}
$$

It follows from the fact that $\Delta(A)(A \otimes 1)=A \otimes A$ and from the bijectivity of the antipode that the algebra $A \# \hat{A}$, as acting on $A$, is precisely the linear span of the operators on $A$ of the form $a^{\prime} \rightarrow \varphi\left(c a^{\prime}\right) a$ with $a, c \in A$. 
It is not hard to obtain the structure of $A \# \hat{A}$ from this. Consider $A \otimes \hat{A}$, together with the product

$$
(a \otimes b)\left(a^{\prime} \otimes b^{\prime}\right)=\left\langle a^{\prime}, b\right\rangle a \otimes b^{\prime}
$$

whenever $a, a^{\prime} \in A$ and $b, b^{\prime} \in \hat{A}$. When $A \otimes \hat{A}$ is considered with this product, we will use $A \diamond \hat{A}$ and for the elements $a \diamond b$. This algebra has an obvious faithful action on $A$ given by

$$
(a \diamond b) a^{\prime}=\left\langle a^{\prime}, b\right\rangle a
$$

whenever $a, a^{\prime} \in A$ and $b \in \hat{A}$. Then the following result is an immediate consequence of proposition 6.5.

6.7 Proposition The map $\gamma: A \# \hat{A} \rightarrow A \diamond \hat{A}$, defined by

$$
\gamma(a \# \varphi(c \cdot))=\sum a S\left(c_{(1)}\right) \diamond \varphi\left(c_{(2)} \cdot\right)
$$

is an isomorphism of algebras.

Remark that the algebra structure of $A \diamond \hat{A}$ no longer depends on the products, nor on the coproducts of $A$ and $\hat{A}$. It only depends on the bilinear form between the two vector spaces $A$ and $\hat{A}$.

In a similar way, we can consider the algebra $\hat{A} \diamond A$. We get an obvious algebra isomorphism of $\hat{A} \# A$ with $\hat{A} \diamond A$.

We also have seen in proposition 6.4 that $A \# \hat{A}$ is anti-isomorphic with $\hat{A} \# A$. It is not hard to see that also $A \diamond \hat{A}$ and $\hat{A} \diamond A$ are anti-isomorphic. The flip will realize such an anti-isomorphism.

In fact, it is also possible to construct an isomorphism of $A \diamond \hat{A}$ to $\hat{A} \diamond A$. Any bijective linear map from $A$ to $\hat{A}$ will do. It is also not so difficult to realize an isomorphism from $A \# \hat{A}$ to $\hat{A} \# A$. Indeed, we know that both algebras act on $A$. We have the natural left action of $A \# \hat{A}$ considered before and given by

$$
(a \# b) a^{\prime}=\sum\left\langle a_{(2)}^{\prime}, b\right\rangle a a_{(1)}^{\prime}
$$

but we also have the right action of $\hat{A} \# A$ on $A$ given by

$$
a^{\prime}(b \# a)=\sum\left\langle a_{(1)}^{\prime}, b\right\rangle a_{(2)}^{\prime} a .
$$

If now, we take $b=\psi(\cdot c)$ with $c \in A$ and a right integral $\psi$ on $A$, we will find

$$
\begin{aligned}
\sum \psi\left(a_{(1)}^{\prime} c\right) a_{(2)}^{\prime} a & =\sum \psi\left(a_{(1)}^{\prime} c_{(1)}\right) a_{(2)}^{\prime} c_{(2)} S\left(c_{(3)}\right) a \\
& =\sum \psi\left(a^{\prime} c_{(1)}\right) S\left(c_{(2)}\right) a
\end{aligned}
$$

As in the proof of 6.6, it follows again that the right action of $\hat{A} \# A$ on $A$ is spanned by the rank one operators from $A$ to $A$ of the form $a^{\prime} \rightarrow \psi\left(a^{\prime} c\right) a$ with $a \in A$ and 
$c \in A$. But also $\hat{A}$ is the set of functionals on $A$ given by $\psi(\cdot c)$ with $c \in A$ and hence, we get again the algebra $A \diamond \hat{A}$.

Let us finish this section with a few remarks.

We have started with a dual pair $(A, B)$ of multiplier Hopf algebras and we have assumed that the pairing is non-degenerate. In the case of a degenerate pairing, similar results can be obtained, but one has to be somewhat more careful.

A particular case is obtained when the algebra $A$ is actually a Hopf algebra. Then, for $B$ one can take a Hopf subalgebra of the dual algebra $A^{\prime}$ of $A$. This is in fact the setting for the duality considered by Montgomery in [M2]. If $B$ is large enough (i.e. separates points), then we have a non-degenerate pairing. Otherwise, this is not the case.

Finally, the finite dimensional case is obviously a special case of the pairing between $A$ and $\hat{A}$ for an algebraic quantum group. Then, the smash product $\hat{A} \# A$ is simply the algebra of $n \times n$ matrices over $\mathbb{C}$ where $n$ is the dimension of $A$. 


\section{Duality}

In this section, we will obtain the main result of this paper. We first define the dual action on the smash product and then we will study the bismash product. We do this for any dual pair of regular multiplier Hopf algebras. However, the nicest duality theorem is obtained for an algebraic quantum group $A$. In this case, the bismash product is an algebra with a very simple structure, no longer depending on the product and the coproduct of $A$, nor on the action of $A$. This situation is similar to (and in fact strongly connected with) the result obtained in proposition 6.6 on the structure of $A \# \hat{A}$. The result that we can prove in the case of a general pairing requires some extra conditions which makes it really a result on coactions. Since we are preparing a separate paper on coactions, we will only state the result here and make some comments. In [VD-Z3], we will treat this result in full detail. It should be noted however that the extra conditions needed in the the case of a general pairing are automatically fulfilled for the pair $(A, \hat{A})$. So the duality theorem that we will prove in [VD-Z3] is an extension of the result that we have here on $(A, \hat{A})$.

So, as before, let $(A, \Delta)$ be a regular multiplier Hopf algebra, acting on an algebra $R$. Consider the smash product $R \# A$ as defined and studied in section 5 . Let $(B, \Delta)$ be another regular multiplier Hopf algebra, paired with $(A, \Delta)$ in the sense of $[\mathrm{D}-\mathrm{VD}]$, as explained in the previous section. Again assume that the pairing is non-degenerate.

Recall that we have a left action of $B$ on $A$ given by

$$
b \triangleright a=\sum\left\langle a_{(2)}, b\right\rangle a_{(1)}
$$

and remark that in this formula, $b$ covers $a_{(2)}$ through the pairing. This left action of $B$ on $A$ gives the dual action of $B$ on $R \# A$ in the following definition.

7.1 Definition Define a linear map from $B \otimes(R \# A)$ to $R \# A$ by

$$
b(x \# a)=x \#(b \triangleright a)=\sum\left\langle a_{(2)}, b\right\rangle x \# a_{(1)}
$$

whenever $a \in A, b \in B$ and $x \in R$.

It easily follows that this map makes $R \# A$ into a left $B$-module. It is a unital module because $A$ is a unital $B$-module. But just as $A$ is a $B$-module algebra, the same is true for $R \# A$ :

7.2 Proposition The action defined above makes $R \# A$ into a $B$-module algebra. 
Proof : Take $a, a^{\prime} \in A, b \in B$ and $x, x^{\prime} \in R$. Then

$$
\begin{aligned}
b\left(\left(x^{\prime} \# a^{\prime}\right)(x \# a)\right) & =\sum b\left(x^{\prime}\left(a_{(1)}^{\prime} x\right) \# a_{(2)}^{\prime} a\right) \\
& =\sum x^{\prime}\left(a_{(1)}^{\prime} x\right) \#\left(b \triangleright\left(a_{(2)}^{\prime} a\right)\right) \\
& =\sum x^{\prime}\left(a_{(1)}^{\prime} x\right) \#\left(b_{(1)} \triangleright a_{(2)}^{\prime}\right)\left(b_{(2)} \triangleright a\right) \\
& =\sum\left(x^{\prime} \#\left(b_{(1)} \triangleright a^{\prime}\right)\right)\left(x \#\left(b_{(2)} \triangleright a\right)\right) \\
& =\sum\left(b_{(1)}\left(x^{\prime} \# a^{\prime}\right)\right)\left(b_{(2)}(x \# a)\right) .
\end{aligned}
$$

This proves that we have a $B$-module algebra.

Remark that we always have the appropriate coverings in the above calculations. We e.g. have that $a_{(1)}^{\prime}$ is covered by $x$ and that $b_{(2)}$ is covered by $a$ or by $x \# a$. We also have used that the left action of $B$ on $A$ commutes with the comultiplication in the sense that

$$
\Delta(b \triangleright a)\left(a^{\prime} \otimes 1\right)=\sum a_{(1)} a^{\prime} \otimes\left(b \triangleright a_{(2)}\right)
$$

for all $a, a^{\prime} \in A$ and $b \in B$.

It is interesting to look at the fixed points in $M(R \# A)$ for the dual action. Remember that we have a homomorphism $\pi: R \rightarrow M(R \# A)$ given by

$$
\pi(x)\left(x^{\prime} \# a^{\prime}\right)=x x^{\prime} \# a^{\prime}
$$

(see proposition 5.8). It is not so difficult to see that $\pi(x)$ is a fixed point. Indeed, if $x, x^{\prime} \in R, a^{\prime} \in A$ and $b \in B$, we get

$$
b\left(\pi(x)\left(x^{\prime} \# a^{\prime}\right)\right)=x x^{\prime} \# b \triangleright a^{\prime}=\pi(x) b\left(x^{\prime} \# a^{\prime}\right)
$$

and this means that $\pi(x)$ is a fixed point (see the remark following proposition 4.11). As we have mentioned already in section 5 (cf. the remark after proposition 5.9 ), we can extend the homomorphism $\pi$ to the multiplier algebra $M(R)$ and the formula is still given by

$$
\pi(m)(x \# a)=m x \# a
$$

whenever $a \in A, x \in R$ and $m \in M(R)$. It is again easy to see that also $\pi(m)$ is a fixed point for the dual action.

In fact, we can prove the converse:

7.3 Proposition The algebra of fixed points in $M(R \# A)$ for the dual action is precisely the image of $M(R)$ under the canonical imbedding $\pi$.

Proof : So, let $z$ be a multiplier of $R \# A$ and assume that $z$ is invariant under the dual action. Fix $x \in R$ and let $\omega \in R^{\prime}$. Define a linear map $\ell: A \rightarrow A$ by

$$
\ell(a)=(\omega \otimes \iota)(z(x \# a)) .
$$


For all $a, a^{\prime} \in A$ we have

$$
\ell\left(a a^{\prime}\right)=(\omega \otimes \iota)\left(z\left(x \# a a^{\prime}\right)\right)=(\omega \otimes \iota)\left(z(x \# a) \pi\left(a^{\prime}\right)\right)
$$

and since $\left(x^{\prime \prime} \# a^{\prime \prime}\right) \pi\left(a^{\prime}\right)=x^{\prime \prime} \# a^{\prime \prime} a^{\prime}$, we get

$$
(\omega \otimes \iota)\left(z(x \# a) \pi\left(a^{\prime}\right)\right)=((\omega \otimes \iota)(z(x \# a))) a^{\prime}
$$

so that $\ell\left(a a^{\prime}\right)=\ell(a) a^{\prime}$. This means that $\ell$ is a left multiplier of $A$. We will write $\ell a$ for $\ell(a)$ in what follows.

Now, we will use that $z$ is fixed under the dual action. This means that $b(z(x \# a))=z(b(x \# a))=z(x \# b \triangleright a)$. Because $b$ acts on the second factor, we get

$$
\begin{aligned}
b \triangleright \ell a & =(\omega \otimes \iota)(b(z(x \# a))) \\
& =(\omega \otimes \iota)(z(x \# b \triangleright a)) \\
& =\ell(b \triangleright a) .
\end{aligned}
$$

So, we see that $\ell$ is a left multiplier on $A$ and fixed under the action of $B$. We have shown in section 6 (remark after proposition 6.5) that this implies that $\ell$ is a scalar multiple of the identity. It follows that there is a number $\lambda$ such that

$$
(\omega \otimes \iota)(z(x \# a))=\lambda a
$$

for all $a \in A$. This implies that for all $a \in A$ and $x \in R$ there exists an element $y \in R$ such that

$$
z(x \# a)=y \# a
$$

We will now show that $y$ does not depend on $a$. Take $a, a^{\prime} \in A$. Choose $e \in A$ so that $e a=a$ and $e a^{\prime}=a^{\prime}$. Take $y$ so that $z(x \# e)=y \# e$. If we multiply this equation with $\pi(a)$ on the right, we find that also $z(x \# a)=y \# a$. Similarly, we get $z\left(x \# a^{\prime}\right)=y \# a^{\prime}$. This implies that $y$ does not depend on $a$.

So, we get a linear map, again denoted by $\ell$ from $R$ to $R$ so that $z(x \# a)=$ $\ell(x) \# a$ for all $a \in A$ and $x \in R$. Using once more that $z$ is a left multiplier, we obtain

$$
\begin{aligned}
\sum \ell(x) a_{(1)} x^{\prime} \# a_{(2)} a^{\prime} & =(\ell(x) \# a)\left(x^{\prime} \# a^{\prime}\right) \\
& =(z(x \# a))\left(x^{\prime} \# a^{\prime}\right) \\
& =z\left((x \# a)\left(x^{\prime} \# a^{\prime}\right)\right) \\
& =\sum \ell\left(x\left(a_{(1)} x^{\prime}\right)\right) \# a_{(2)} a^{\prime}
\end{aligned}
$$

for all $a, a^{\prime} \in A$ and $x, x^{\prime} \in R$. If we apply $\epsilon$ to the second factor, we get

$$
\ell\left(x\left(a x^{\prime}\right)\right)=\ell(x) a x^{\prime}
$$


for all $a \in A$ and $x, x^{\prime} \in R$. Hence we have also $\ell\left(x x^{\prime}\right)=\ell(x) x^{\prime}$ so that $\ell$ is a left multiplier of $R$. We will again in what follows denote $\ell(x)$ by $\ell x$.

Now, we will use the fact that $z$ is also a right multiplier to prove that also $\ell$ is a right multiplier. For all $a \in A, x \in R$ and $y \in R \# A$ we get

$$
(y z)(x \# a)=y(\ell x \# a)
$$

Take for $y$ an element of the form $\pi\left(a^{\prime}\right) \pi\left(x^{\prime}\right)$ with $a^{\prime} \in A$ and $x^{\prime} \in R$ and write

$$
\pi\left(a^{\prime}\right) \pi\left(x^{\prime}\right) z=\sum \pi\left(a_{i}\right) \pi\left(x_{i}\right)
$$

to get

$$
\pi\left(a^{\prime}\right) \pi\left(x^{\prime}(\ell x)\right) \pi(a)=\sum \pi\left(a_{i}\right) \pi\left(x_{i} x\right) \pi(a) .
$$

We can cancel $\pi(a)$ and use the injectivity of the map $x \otimes a \rightarrow \pi(a) \pi(x)$ on $R \otimes A$ (because $\left.\pi(a) \pi(x)=\sum a_{(1)} x \# a_{(2)}\right)$ to obtain

$$
x^{\prime}(\ell x) \otimes a^{\prime}=\sum x_{i} x \otimes a_{i}
$$

Now apply any linear functional on the second factor. Then we get for every element $x^{\prime} \in R$ and element $y$ in $R$ satisfying $x^{\prime}(\ell x)=y x$ for all $x \in R$. This implies that $\ell$ is in fact a two-sided multiplier. This completes the proof.

Similar results are known for actions of locally compact quantum groups on operator algebras (see e.g. proposition 4.12 in [VD1] for one of them).

Now, we consider the bismash product $(R \# A) \# B$. The product of two elements $(x \# a) \# b$ and $\left(x^{\prime} \# a^{\prime}\right) \# b^{\prime}$ in $(R \# A) \# B$ is given by

$$
\begin{aligned}
((x \# a) \# b)\left(\left(x^{\prime} \# a^{\prime}\right) \# b^{\prime}\right) & =\sum\left((x \# a)\left(x^{\prime} \#\left(b_{(1)} \triangleright a^{\prime}\right)\right)\right) \# b_{(2)} b^{\prime} \\
& =\sum\left\langle a_{(2)}^{\prime}, b_{(1)}\right\rangle\left((x \# a)\left(x^{\prime} \# a_{(1)}^{\prime}\right)\right) \# b_{(2)} b^{\prime} \\
& =\sum\left\langle a_{(2)}^{\prime}, b_{(1)}\right\rangle\left(\left(x\left(a_{(1)} x^{\prime}\right)\right) \# a_{(2)} a_{(1)}^{\prime}\right) \# b_{(2)} b^{\prime} .
\end{aligned}
$$

Remark that in this last expression, $b^{\prime}$ covers $b_{(2)}$ and $x^{\prime}$ covers $a_{(1)}$. This implies that indirectly, also both $a_{(1)}^{\prime}$ and $a_{(2)}^{\prime}$ are covered.

We have seen that, in general, $R$ can be made into a left $(R \# A)$-module (cf. section $5)$. Here, we can make $R \# A$ into a left $((R \# A) \# B)$-module. We have the following formula. Whenever $(x \# a) \# b$ is an element in $(R \# A) \# B$ and $x^{\prime} \# a^{\prime}$ is in $R \# A$, we get

$$
\begin{aligned}
((x \# a) \# b)\left(x^{\prime} \# a^{\prime}\right) & =(x \# a)\left(x^{\prime} \#\left(b \triangleright a^{\prime}\right)\right) \\
& =\sum\left\langle a_{(2)}^{\prime}, b\right\rangle(x \# a)\left(x^{\prime} \# a_{(1)}^{\prime}\right) \\
& =\sum\left\langle a_{(2)}^{\prime}, b\right\rangle x\left(a_{(1)} x^{\prime}\right) \# a_{(2)} a_{(1)}^{\prime} .
\end{aligned}
$$


Remark again that $a_{(1)}$ is covered by $x^{\prime}$ and $a_{(2)}^{\prime}$ is covered by $b$.

Just as in the case of the action of $A \# B$ on $A$ (see proposition 6.2), also here this action turns out to be faithful:

7.4 Proposition $R \# A$ is a faithful $((R \# A) \# B)$-module for the action defined above.

Proof : Assume that $\sum\left(x_{i} \# a_{i}\right) \# b_{i}$ is in $(R \# A) \# B$ and that

$$
\left(\sum\left(x_{i} \# a_{i}\right) \# b_{i}\right)\left(x^{\prime} \# a^{\prime}\right)=0
$$

for all $x^{\prime} \in R$ and $a^{\prime} \in A$. Then, using the above formula, we get

$$
\sum\left\langle a_{(2)}^{\prime}, b_{i}\right\rangle x_{i}\left(a_{i(1)} x^{\prime}\right) \# a_{i(2)} a_{(1)}^{\prime}=0
$$

for all $x^{\prime} \in R$ and $a^{\prime} \in A$. As in the proof of 6.2 , multiply the second factor in this smash product with $a^{\prime \prime}$ from the right and use that $\Delta(A)(A \otimes 1)=A \otimes A$ to get

$$
\sum\left\langle a^{\prime}, b_{i}\right\rangle x_{i}\left(a_{i(1)} x^{\prime}\right) \# a_{i(2)} a^{\prime \prime}=0
$$

for all $x^{\prime} \in R$ and $a^{\prime}, a^{\prime \prime} \in A$. Then we can use the non-degeneracy of the product in $R \# A$ to obtain that

$$
\sum\left\langle a^{\prime}, b_{i}\right\rangle x_{i} \# a_{i}=0
$$

for all $a^{\prime} \in A$. Finally, because we assume the pairing to be non-degenerate, we get $\sum\left(x_{i} \# a_{i}\right) \# b_{i}=0$.

Now, we will construct an equivalent module. We will denote the underlying space again by $R \otimes A$. Define a linear map $W: R \otimes A \rightarrow R \# A$ by

$$
W(x \otimes a)=\sum a_{(1)} x \# a_{(2)}
$$

This is well-defined because $a_{(1)}$ is covered by $x$. Remark that $W$ is surjective because $\Delta(A)(A \otimes 1)=A \otimes A$ and $R$ is unital. In fact, $W$ is a bijection and the inverse is given by

$$
W^{-1}(x \# a)=\sum S^{-1}\left(a_{(1)}\right) x \otimes a_{(2)}
$$

whenever $a \in A$ and $x \in R$. We have used a similar map in section 5 . In fact, it is the twisting map defining the commutation rules between $\pi(A)$ and $\pi(R)$ (cf. proposition 5.10).

Now, we can use this vector space isomorphism $W$ to construct a new action of $(R \# A) \# B$ on $R \otimes A$. It will still be faithful and we will use it to examine the bismash product $(R \# A) \# B$.

In the next proposition, we obtain the formulas giving this action. 
7.5 Proposition For $a, a^{\prime} \in A, b \in B$ and $x, x^{\prime} \in R$, we get

$$
\begin{aligned}
W^{-1}(x \# a) W\left(x^{\prime} \otimes a^{\prime}\right) & =\sum\left(\left(S^{-1} a_{(1)}^{\prime}\right)\left(S^{-1} a_{(1)}\right) x\right) x^{\prime} \otimes a_{(2)} a_{(2)}^{\prime} \\
W^{-1} b W\left(x^{\prime} \otimes a^{\prime}\right) & =x^{\prime} \otimes\left(b \triangleright a^{\prime}\right) .
\end{aligned}
$$

Proof : We have

$$
\begin{aligned}
W^{-1}(x \# a) W\left(x^{\prime} \otimes a^{\prime}\right) & =W^{-1} \sum(x \# a)\left(a_{(1)}^{\prime} x^{\prime} \# a_{(2)}^{\prime}\right) \\
& =W^{-1} \sum x\left(a_{(1)} a_{(1)}^{\prime} x^{\prime}\right) \# a_{(2)} a_{(2)}^{\prime} \\
& =\sum S^{-1}\left(a_{(2)} a_{(2)}^{\prime}\right)\left(x\left(a_{(1)} a_{(1)}^{\prime} x^{\prime}\right)\right) \otimes a_{(3)} a_{(3)}^{\prime} \\
& =\sum\left(S^{-1}\left(a_{(1)} a_{(1)}^{\prime}\right) x\right) x^{\prime} \otimes a_{(2)} a_{(2)}^{\prime}
\end{aligned}
$$

and

$$
\begin{aligned}
W^{-1} b W\left(x^{\prime} \otimes a^{\prime}\right) & =W^{-1} \sum a_{(1)}^{\prime} x^{\prime} \#\left(b \triangleright a_{(2)}^{\prime}\right) \\
& =W^{-1} \sum\left\langle a_{(3)}^{\prime}, b\right\rangle a_{(1)}^{\prime} x^{\prime} \# a_{(2)}^{\prime} \\
& =\sum\left\langle a_{(2)}^{\prime}, b\right\rangle x^{\prime} \otimes a_{(1)}^{\prime} .
\end{aligned}
$$

If we combine these two formulas, we get

$$
W^{-1}((x \# a) \# b) W\left(x^{\prime} \otimes a^{\prime}\right)=\sum\left\langle a_{(3)}^{\prime}, b\right\rangle\left(\left(S^{-1} a_{(1)}^{\prime}\right)\left(S^{-1} a_{(1)}\right) x\right) x^{\prime} \otimes a_{(2)} a_{(2)}^{\prime}
$$

for all $a, a^{\prime} \in A, b \in B$ and $x, x^{\prime} \in R$.

We are interested in the algebra of linear maps on $R \otimes A$ spanned by such operators. For convenience, we will call this algebra $P$ in what follows.

Now, we have seen that the map

$$
x \otimes a \rightarrow \sum S^{-1}\left(a_{(1)}\right) x \otimes a_{(2)}
$$

is a bijection of $R \otimes A$ (in fact, it is the map $W^{-1}$ ). It follows that the algebra $P$ is the span of all operators of the form

$$
x^{\prime} \otimes a^{\prime} \rightarrow \sum\left\langle a_{(3)}^{\prime}, b\right\rangle\left(\left(S^{-1} a_{(1)}^{\prime}\right) x\right) x^{\prime} \otimes a a_{(2)}^{\prime}
$$

where still $a \in A, b \in B$ and $x \in R$. We can recognise the action of $A \# B$ on $A$ in the last factor. Indeed, the above operator can be rewritten as

$$
x^{\prime} \otimes a^{\prime} \rightarrow \sum\left(\left(S^{-1} a_{(1)}^{\prime}\right) x\right) x^{\prime} \otimes(a \# b) a_{(2)}^{\prime} .
$$

We will come back to this expression later. 
Now, we first want to specialize to the case of an algebraic quantum group.

So, in what follows, let $A$ be an algebraic quantum group and let $B$ be the dual $\hat{A}$ of $A$. Of course, we consider the pairing given by the duality. We have seen in the previous section that the algebra $A \# \hat{A}$, as acting on $A$ is the span of rank one operators of the form $a^{\prime} \rightarrow\left\langle a^{\prime}, b\right\rangle a$ where $a \in A$ and $b \in \hat{A}$ (see proposition 6.6). Remember that we have used the notation $A \diamond \hat{A}$ for this algebra of finite rank operators.

We will now use the same technique as in the proof of proposition 6.6 to obtain the following duality theorem.

7.6 Theorem If $A$ is an algebraic quantum group, acting on an algebra $R$ and if $\hat{A}$ is the dual of $A$, acting on the smash product $R \# A$ by means of the dual action, then the bismash product $(R \# A) \# \hat{A}$ is isomorphic with $R \otimes(A \diamond \hat{A})$.

Proof : Let $a \in A$ and $b \in \hat{A}$. Write $b$ in the form $\varphi(c \cdot)$ where $\varphi$ is a left integral on $A$ and $c \in A$. As we had in the proof of proposition 6.6 , we get

$$
\sum\left\langle a_{(2)}^{\prime}, b\right\rangle a_{(1)}^{\prime}=\sum \varphi\left(c_{(2)} a^{\prime}\right) S\left(c_{(1)}\right) .
$$

So, we find that the algebra $P$ is spanned by operators of the form

$$
x^{\prime} \otimes a^{\prime} \rightarrow \sum \varphi\left(c_{(3)} a^{\prime}\right)\left(c_{(2)} x\right) x^{\prime} \otimes a S\left(c_{(1)}\right)
$$

where $a, c \in A$ and $x \in R$. Because $(A \otimes 1) \Delta(A)=A \otimes A$, we get all operators of the form

$$
x^{\prime} \otimes a^{\prime} \rightarrow \sum \varphi\left(c_{(2)} a^{\prime}\right)\left(c_{(1)} x\right) x^{\prime} \otimes a .
$$

and by the fact that

$$
x \otimes c \rightarrow \sum c_{(1)} x \otimes c_{(2)}
$$

is surjective on $R \otimes A$, we have that $P$ is spanned by operators of the form

$$
x^{\prime} \otimes a^{\prime} \rightarrow x x^{\prime} \otimes \varphi\left(c a^{\prime}\right) a,
$$

still with $x \in R$ and $a, c \in A$. Since we are dealing with faithful actions of these two algebras, we get an isomorphism. This completes the proof.

If we examine the proof more carefully and if we follow the different steps in it, we can find an explicit expression for the isomorphism of $(R \# A) \# \hat{A}$ to $R \otimes(A \diamond \hat{A})$. Of course, it would be possible to prove theorem 7.6 by using this map and showing that it is actually an algebra isomorphism. However, it is clear that the calculations will become very involved. On the other hand, the technique we used here, by considering a faithful action of our algebra, not only makes the proof easier, but it also shows where the isomorphism comes from. 
As a special case of this duality for actions of algebraic quantum groups, we get of course the duality for actions of finite-dimensional Hopf algebras ([vdB], see also [M2, page 167]). Indeed, every finite-dimensional Hopf algebra is an algebraic quantum group and the dual is precisely the dual Hopf algebra.

Now, we turn our attention again to the case of a general pairing. For this, let us look again at the statement, preceding theorem 7.6. It says that the algebra $P$ (which is isomorphic with the bismash product) is spanned by the operators on $R \otimes A$ of the form

$$
x^{\prime} \otimes a^{\prime} \rightarrow \sum\left(\left(S^{-1} a_{(1)}^{\prime}\right) x\right) x^{\prime} \otimes(a \# b) a_{(2)}^{\prime}
$$

where in the last factor, we use the natural left action of $B \# A$ on $A$.

If we examine the above formula, we see that we have some kind of a twisting of the algebras $R$ and $A \# B$. The action of $R$ on $R \otimes A$ here is given by

$$
x^{\prime} \otimes a^{\prime} \rightarrow \sum\left(\left(S^{-1} a_{(1)}^{\prime}\right) x\right) x^{\prime} \otimes a_{(2)}^{\prime}
$$

In the case $(A, \hat{A})$, it is essentially due to the fact that $A \# \hat{A}$ acts by means of finite rank operators on $A$, that we are able to unfold the effect of this twisting. In the general case we realize this untwisting by a completely different technique. We add a condition on the $A$-module $R$ to ensure that $A x$ does not become too big (finite-dimensional for the case where $A$ is an algebra with 1) for any $x \in R$.

We need the notion of a coaction (see [VD-Z3]).

7.7 Definition If $R$ is an algebra over $\mathbb{C}$ and if $B$ is a regular multiplier Hopf algebra, then a coaction of $B$ on $R$ is an injective homomorphism $\Gamma: R \rightarrow$ $M(R \otimes B)$ such that $\Gamma(R)(1 \otimes B) \subseteq R \otimes B$ and $(1 \otimes B) \Gamma(R) \subseteq R \otimes B$ and such that $(\Gamma \otimes \iota) \Gamma=(\iota \otimes \Delta) \Gamma$.

This last form of coassociativity can be given a meaning here in different ways. We can e.g. write it as

$$
(\Gamma \otimes \iota)(\Gamma(x)(1 \otimes b))=(\iota \otimes \Delta)(\Gamma(x))(1 \otimes 1 \otimes b)
$$

Indeed, because $\Gamma(x)(1 \otimes b) \in R \otimes B$, the left hand side makes sense, while for the right hand side, just observe that $\Delta$ is non-degenerate so that $\iota \otimes \Delta$ can be extended to the multiplier algebra $M(R \otimes B)$.

It can be shown that for all coactions, we have the equalities $\Gamma(R)(1 \otimes B)=R \otimes B$ and $(1 \otimes B) \Gamma(R)=R \otimes B$. In fact, the maps

$$
\begin{aligned}
& x \otimes b \rightarrow \Gamma(x)(1 \otimes b) \\
& x \otimes b \rightarrow(1 \otimes b) \Gamma(x)
\end{aligned}
$$


are injective on $R \otimes B$ (see [VD-Z3]).

A trivial example of a coaction is the coaction of $B$ on itself, given by the comultiplication $\Delta$. The bijectivity of the maps above are in this case multiplier Hopf algebra axioms.

It is not hard to see that any coaction of $B$ on $R$ will yield an action of $A$ on $R$ by means of the formula

$$
a x=\left(\iota \otimes \omega_{a}\right) \Gamma(x)
$$

where $\omega_{a}$ denotes the linear functional on $B$ given by $b \rightarrow\langle a, b\rangle$. Remark, when $a=b^{\prime} \triangleright a^{\prime}$, we see that

$$
\left(\iota \otimes \omega_{a}\right) \Gamma(x)=\left(\iota \otimes \omega_{a}^{\prime}\right)\left(\Gamma(x)\left(1 \otimes b^{\prime}\right)\right)
$$

and this is well-defined. Hence, because the action of $B$ on $A$ is unital, also $(\iota \otimes$ $\left.\omega_{a}\right) \Gamma(x)$ makes sense. Because of the fact that e.g. $\Gamma(R)(1 \otimes B)=R \otimes B$, this associated action is unital.

Not every action of $A$ will come from a coaction of $B$. However, if $A$ is an algebraic quantum group and if $B=\hat{A}$, then this is the case. So, for algebraic quantum groups, there is a one-to-one correspondence between the actions of $A$ and the coactions of $\hat{A}$ (see [VD-Z3]).

Now, let us go back to the bismash product $(R \# A) \# B$ for a general pair $(A, B)$. Assume that the action of $A$ on $R$ comes from a coaction $\Gamma$ of $B$ on $R$. Let us use the Sweedler notation also for these coactions. So, write $\Gamma(x)=\sum x_{(0)} \otimes x_{(1)}$ and make sure that $x_{(1)}$ is always covered by an element of $B$. Then the algebra $P$ of operators on $R \otimes A$ considered before, is spanned by linear operators of the form

$$
x^{\prime} \otimes a^{\prime} \rightarrow \sum x_{(0)} x^{\prime} \otimes\left\langle S^{-1} a_{(1)}^{\prime}, x_{(1)}\right\rangle(a \# b) a_{(2)}^{\prime} .
$$

In this formula, the element $a_{(2)}^{\prime}$ is covered by $a \# b$ and so, also $x_{(1)}$ will be covered through the pairing with $S^{-1} a_{(1)}^{\prime}$ and this expression has a meaning.

It is seen from the above formula that $P$ will be a subalgebra of the tensor product algebra $R \otimes Q$ where of course, $R$ acts on $R$ by left multiplication and where $Q$ is the algebra generated by the left action of $A \# B$ on $A$ on the one hand, and by the right action of $B$ on $A$ on the other hand.

So, we clearly need some extra condition. It is similar to the RL-condition as found in 9.4.5 of [M2]. In this context, we require that the right action of an element $b \in B$ on $A$ is a multiplier of the algebra $A \# B$ as acting on $A$. In other words, the algebra $Q$ above coincides with the algebra $A \# B$ as acting on $A$.

There are some more arguments needed, but at least, this explains in some sense the result that we get in [VD-Z3] and that is formulated in the next theorem.

7.8 Theorem If the action of $A$ on $R$ comes from a coaction of $B$ and if the pairing between $A$ and $B$ is such that the right action of $B$ on $A$ is in the 
multiplier algebra of $A \# B$, as acting also on $A$, then, $(R \# A) \# B$ is isomorphic with $R \otimes(A \# B)$.

This theorem is the natural generalization of the duality theorem for actions of Hopf algebras as obtained by Blattner and Montgomery ([B-M], [M2]) to the multiplier Hopf algebra case. The proof that we will give in [VD-Z3] will use similar techniques as we have used to obtain theorem 7.6 and so, it is also slightly simpler that the one in [M2]. Also remark that the extra condition on the pairing is always fulfilled for the pair $(A, \hat{A})$. Because also every action of $A$ comes from a coaction of $\hat{A}$ in this case, theorem 7.8 also generalizes theorem 7.6.

The two duality theorems 7.6 and 7.8 can be combined with the fixed point result in proposition 7.3 as follows. Consider the bidual action of $A$ on the bismash product $(R \# A) \# B$. Use the isomorphism with $R \otimes(A \# B)$ to transform the bidual action to an action of $A$ on $R \otimes(A \# B)$. The multiplier algebra of $R \# A$ can be obtained as a fixed point algebra. One could also try to use this idea to view the smash product $R \# A$ itself as some kind of a fixed point algebra. Similar techniques have been used in the theory of crossed products of operator algebras by actions of locally compact groups to give such a characterization of the crossed product in such a case (see e.g. theorem 3.11 in [VD1]).

\section{REFERENCES}

[A] E. Abe, Hopf algebras, Cambridge University Press, 1977.

[B-M] R. Blattner \& S. Montgomery, A duality theorem for Hopf module algebras, J. Algebra 95 (1985), 153-172.

[B-S] S. Baaj \& G. Skandalis, Unitaires multiplicatifs et dualité pour les produits croisés de $C^{*}$-algèbres., Ann. Scient. Ec. Norm. Sup., 4ème série 26 (1993), 425-488.

[C-M] M. Cohen \& S. Montgomery, Group-graded rings, smash products and group actions, Trans. Amer. Math. Soc. 282 (1984), 237-258.

[D-VD] B. Drabant \& A. Van Daele, Pairing and Quantum Double of Multiplier Hopf Algebras, Preprint K.U. Leuven (1996).

[D] V.G. Drinfel'd, Quantum groups, Proceedings of the ICM, Berkeley, 1986, pp. 798-820.

[E-R] E.G. Effros \& Z.-J. Ruan, Discrete quantum groups I. The Haar measure, Int. J. Math. 5 (1994), 681-723.

[E-S] M. Enock \& J.-M. Schwartz, Kac algebras and duality for locally compact groups, Springer, 1992.

[K-VD] J. Kustermans \& A. Van Daele, $C^{*}$-algebraic quantum groups arising from algebraic quantum groups, Int. J. Math. 8, No. 8 (1997), 1067-1139.

[K] J. Kustermans, $C^{*}$-algebraic quantum groups arising from algebraic quantum groups, Ph.D. thesis K.U. Leuven (1997).

[L] M. Landstad, Duality for covariant systems, Trans. Amer. Math. Soc. 248 (1979), 223-267.

[M1] S. Montgomery, Duality for actions and coactions of groups, Contemporary Mathematics 43 (1985), 191-207.

[M2] S. Montgomery, Hopf algebras and their actions on rings, vol. 82, CBMS notes, AMS, Providence, 1993.

[M-N] M. Masuda \& Y. Nakagami, A von Neumann algebra framework for the duality of quantum groups, Publ. of the RIMS 30 (1994), Kyoto, 799-850.

[M-N-W] M. Masuda, Y. Nakagami \& S. Woronowicz, (in preparation).

[Ng] C.-K. Ng, Coactions and crossed products of Hopf $C^{*}$-algebras, Proc. London Math. Soc. 72 (1996), 638-656. 
[N-T] Y. Nakagami \& M Takesaki, Duality for crossed products of von Neumann algebras, Lecture Notes in Mathematics, Springer, 1979, pp. 731.

[P] G.K. Pedersen, C*-algebras and their automorphism groups, Academic Press, 1979.

[Q] D. Quinn, Group-graded rings and duality, Trans. Amer. Math. Soc. 292 (1985), 155167.

[Sa] S. Sakai, $C^{*}$-algebras and $W^{*}$-algebras, Springer, 1971.

[Sw] M.E. Sweedler, Hopf algebras, Benjamin, New York, 1969.

[T] H. Takai, On duality for crossed products of $C^{*}$-algebras, J. Funct. Anal. 19 (1975), 25-39.

[Ts] M. Takesaki, Duality and the structure of von Neumann algebras of type III, Acta Math. 131 (1973), 249-310.

[VD1] A. Van Daele, Continuous crossed products and type III von Neumann algebras, vol. 31, London Math. Soc. Lecture Note Series, Cambridge University Press, 1978.

[VD2] _ Dual pairs of Hopf ${ }^{*}$-algebras, Bull. London Math. Soc. 25 (1993), 209-230.

[VD3] ㄴ Multiplier Hopf algebras, Trans. Amer. Math. Soc. 342 (1994), 917-932.

[VD4] _ Discrete quantum groups, J. of Algebra 180 (1996), 431-444.

[VD5] - Multiplier Hopf algebras and duality, Quantum Groups and Quantum Spaces, vol. 40, Warsaw, 1997, pp. 51-58.

[VD6] _ An algebraic framework for group duality, Preprint University of Oslo. To appear in Advances of Mathematics (1996).

[VD-VK] A. Van Daele \& S. Van Keer, The Yang-Baxter and Pentagon equation, Comp. Math. 91 (1994), 201-221.

[VD-Z1] A. Van Daele \& Y. Zhang, Multiplier Hopf algebras of discrete type, Preprint K.U. Leuven (1996).

[VD-Z2] Galois theory for multiplier Hopf with integrals, Preprint K.U. Leuven (1997).

[VD-Z3] Coactions for multiplier Hopf algebras, (In preparation).

[vdB] M. van den Bergh, A duality theorem for Hopf algebras, Methods in Ring Theory, Nato ASI series 129, (1984), Reidel, Dordrecht, 517-522.

[W1] S.L. Woronowicz, Compact matrix pseudogroups, Comm. Math. Physics 111 (1987), 613-665.

[W2] C Compact quantum groups, Preprint University of Warsaw (1992).

Drabant: DAMTP, University of Cambridge, Silver Street, Cambridge CB3 9EW, UK Van Daele and Zhang: Department of Mathematics, KU Leuven, Celestijnenlaan 200B, B-3001 Heverlee, Belgium 